ARTICLE

\title{
LRRK2 G2019S mutation attenuates microglial motility by inhibiting focal adhesion kinase
}

Insup Choi1,2,3, Beomsue Kim², Ji-Won Byun²,2, Sung Hoon Baik ${ }^{4}$, Yun Hyun Huh5, Jong-Hyeon Kim², Inhee Mook-Jung ${ }^{4}$, Woo Keun Song ${ }^{5}$, Joo-Ho Shin ${ }^{6}$, Hyemyung Seo ${ }^{7}$, Young Ho Suh ${ }^{1,2,3}$, Ilo Jou ${ }^{1,2,3}$, Sang Myun Park ${ }^{1,2,3}$, Ho Chul Kang ${ }^{8}$ \& Eun-Hye Joe $1,2,3,9,10$

In response to brain injury, microglia rapidly extend processes that isolate lesion sites and protect the brain from further injury. Here we report that microglia carrying a pathogenic mutation in the Parkinson's disease (PD)-associated gene, G2019S-LRRK2 (GS-Tg microglia), show retarded ADP-induced motility and delayed isolation of injury, compared with non-Tg microglia. Conversely, LRRK2 knockdown microglia are highly motile compared with control cells. In our functional assays, LRRK2 binds to focal adhesion kinase (FAK) and phosphorylates its Thr-X-Arg/Lys (TXR/K) motif(s), eventually attenuating FAK activity marked by decreased pY397 phosphorylation (pY397). GS-LRRK2 decreases the levels of pY397 in the brain, microglia and HEK cells. In addition, treatment with an inhibitor of LRRK2 kinase restores pY397 levels, decreased pTXR levels and rescued motility of GS-Tg microglia. These results collectively suggest that G2019S mutation of LRRK2 may contribute to the development of PD by inhibiting microglial response to brain injury.

\footnotetext{
${ }^{1}$ Department of Biomedical Sciences, Neuroscience Graduate Program, Ajou University School of Medicine, Suwon, Gyeonggi-do 443-380, Korea.

${ }^{2}$ Department of Pharmacology, Ajou University School of Medicine, Suwon, Gyeonggi-do 443-380, Korea. ${ }^{3}$ Chronic Inflammatory Disease Research Center, Ajou University School of Medicine, Suwon, Gyeonggi-do 443-380, Korea. ${ }^{4}$ Department of Biochemistry and Biomedical Sciences, College of Medicine, Seoul National University, Seoul 110-799, Korea. ${ }^{5}$ Bio Imaging and Cell Dynamics Research Center, School of Life Sciences, Gwangju Institute of Science and Technology, Gwangju 500-712, Korea. ${ }^{6}$ Division of Pharmacology, Department of Molecular Cell Biology, Samsung Biomedical Research Institute, Sungkyunkwan University School of Medicine, Suwon, Gyeonggi-do 440-746, Korea. ${ }^{7}$ Department of Molecular and Life Sciences, Hanyang University, Ansan 426-791, Korea. ${ }^{8}$ Department of Physiology, Ajou University School of Medicine, Suwon, Gyeonggi-do 443-380, Korea. ${ }^{9}$ Department of Brain Science, Ajou University School of Medicine, Suwon, Gyeonggi-do 443-380, Korea. ${ }^{10}$ Brain Disease Research Center, Ajou University School of Medicine, Suwon, Gyeonggi-do 443-380, Korea. Correspondence and requests for materials should be addressed to E.-H.J. (email: ehjoe@ajou.ac.kr).
} 
eucine-rich repeat kinase 2 (LRRK2) is an autosomal dominant, late-onset familial Parkinson's disease (PD) gene. LRRK2 consists of several functional domains, including armadillo, ankyrin-like, leucine-rich repeat, Ras of complex proteins, C-terminal of Ras of complex proteins, kinase and WD40 domains, which may support diverse functions of LRRK2 (ref. 1). An important role of LRRK2 is regulation of actin dynamics; LRRK2 interacts with several actin-regulatory proteins, and regulates filopodia protrusion and neurite outgrowth ${ }^{2-5}$. PD-associated pathogenic mutations in LRRK2 are scattered throughout the entire $L R R K 2$ gene ${ }^{6}$. Of the mutations identified to date, G2019S has received the most attention since it is also found in sporadic $\mathrm{PD}^{7,8}$. G2019S is a pathogenic gain-of-function mutant that exhibits increased kinase activity, and thereby attenuates neurite outgrowth and increases neuronal death ${ }^{9-11}$.

Microglia, brain macrophages, use their highly branched processes to continuously scan the entire brain environment for abnormal structures and injury ${ }^{12-14}$. In response to brain injury, microglia rapidly extend processes to isolate lesion sites, preventing further injury that might be produced by disruptions in microenvironmental homeostatic ${ }^{12,13,15-17}$. Accordingly, it has been reported that, in the presence of an actin-depolymerizing agent, microglia do not properly isolate injury sites, worsening the damage ${ }^{15}$. Furthermore, defects in microglia have been reported in neurodegenerative diseases, including Alzheimer's disease and Huntington's disease ${ }^{18,19}$. In aged brains, microglial motility is also slowed ${ }^{20,21}$. Although microglia express LRRK2 (refs 22,23), whether LRRK2 regulates microglial motility has not been studied.

Focal adhesion kinase (FAK) is a non-receptor tyrosine kinase that affects a range of cellular functions, including migration, proliferation, and survival ${ }^{24-26}$. FAK consists of an N-terminal FERM domain, a tyrosine kinase domain and a C-terminal focal adhesion targeting (FAT) domain ${ }^{27,28}$. It was originally reported that FAK is activated by the interaction between extracellular matrix and integrin ${ }^{29,30}$. FAK is also activated by several external stimuli, including activation of growth factor receptors or $G$ protein-coupled receptors, and mechanical stress $^{31-34}$. Although FAK is phosphorylated at multiple sites in response to stimuli, Y397 phosphorylation (pY397) is important for the proper integration of signalling pathways that control cell adhesion and migration ${ }^{26,35}$.

In this study, we report that LRRK2 is a negative regulator of microglial motility. LRRK2 inhibits FAK activation in a kinase-dependent manner, meaning that the G2019S gain-offunction mutation results in the excessive inhibition of FAK activation and microglial motility. Collectively, our findings suggest that the G2019S mutation of LRRK2 may prevent microglia from efficiently responding to brain damage, thereby contributing to the development of PD.

\section{Results}

The LRRK2 G2019S mutation retards microglial motility. In keeping with their role in continually surveying the brain microenvironment ${ }^{12,14}$, microglia have been reported to stretch their processes towards injured sites in response to purines, including ADP, ATP and UDP, released from damaged cells ${ }^{13,36}$. Since LRRK2 regulates actin dynamics $2,4,5$, we examined whether LRRK2 regulates microglial motility using microglia cultured from brain of G2019S-LRRK2 transgenic (Tg) mice and littermate non-Tg mice. The morphologies of non-Tg and GS-Tg microglia were similarly diverse and indistinguishable (Fig. 1a). Non-Tg microglia rapidly (within $5 \mathrm{~min}$ ) responded to ADP by forming lamellipodia (black arrowheads in Fig. 1a), a marker of migrating cells, and moving cell bodies for about $20 \mathrm{~min}$
(Fig. 1a; Supplementary Movie 1). Interestingly, however, GS-Tg microglia formed lamellipodia that rapidly shrunk by $10-15 \mathrm{~min}$ (white arrowheads in Fig. 1a). Furthermore, GS-Tg microglia barely moved in response to ADP (Fig. 1a; Supplementary Movie 1). Quantitative analyses using stroboscopic analysis of cell dynamics (SACED) showed that in response to ADP, GS-Tg microglia produced short protrusions $(p)$ that were immediately retracted $(r)$, whereas non-Tg cells exhibited long and wide protrusions $(p)$ that rarely retracted $(r)$ (Fig. 1b,c). In addition, we measured cell size reflecting the extent of lamellipodia protrusion from non-Tg and GS-Tg microglia in response to ADP. Consistent with SACED analysis, GS-Tg microglia exhibited significant decreased cell size from 5 to $20 \mathrm{~min}$ compared with non-Tg microglia (Supplementary Fig. 1), suggesting that GS-Tg microglia do not form lamellipodia in the presence of ADP. In migration assays using Transwells, GS-Tg microglia exhibited retarded migration compared with non-Tg microglia (Fig. 1d). On the basis of a previous report that ADP induces the movement of microglia through $\mathrm{P}_{2} \mathrm{Y}_{12}$ receptor ${ }^{36}$, we further used RT-PCR and immunostaining to examine $\mathrm{P}_{2} \mathrm{Y}_{12}$ receptor levels in non-Tg and GS-Tg microglia. However, we observed little difference in this parameter (Supplementary Fig. 2).

Next, we examined whether LRRK2 knockdown (KD) affected the morphology and motility of microglia by comparing LRRK2-KD BV2 microglia and control cells prepared using $L R R K 2$-targeted and non-targeted (NT) small hairpin RNAs (shRNAs), respectively ${ }^{22}$. Two different LRRK2-KD clones were tested and both showed a morphology typical of migrating cells flat and polarized (white and black arrowheads), and firmly attached to the substratum, whereas NT cells were weakly attached to the substratum and harboured short processes with relatively round shapes (Fig. 1e). LRRK2-KD cells stained with Alexa-488 phalloidin showed a dense F-actin structure at the leading edge compared with NT cells (Fig. 1f). Furthermore, LRRK2-KD cells moved much faster than NT cells, even in the absence of any activators (Fig. 1g). 'Chase' experiments, in which individual NT and LRRK2-KD cells were followed for $6 \mathrm{~h}$, revealed the obviously more rapid movement of LRRK2-KD cells (Fig. 1h; Supplementary Movie 2). Moving velocities of NT cells and LRRK2-KD cells were $7.5 \pm 0.4 \times 10^{-3} \mu \mathrm{m} \mathrm{s}^{-1}$ and $14.5 \pm 1.5 \times 10^{-3} \mu \mathrm{m} \mathrm{s}^{-1}$ (mean \pm s.e.m. of 15 cells), respectively. These data suggest that LRRK2 negatively regulate microglia motility in vitro.

Since microglia rapidly respond to injury and isolate damaged sites $^{13,15}$, we used stab-wound and laser-induced injury models to examine the microglial response in non-Tg and GS-Tg mice. In the stab-wound injury model, Iba-1-positive microglia rapidly surrounded the injury sites within $1 \mathrm{~h}$ regardless of the tested genotype (Fig. 2a). However, microglia in GS-Tg mice isolated the injury sites less tightly compared with those in non-Tg mice (see 2 and 4 in Fig. 2a). The Image J software was used to quantify the Iba-1-positive pixels around the injury sites (Fig. 2b, upper panel), and the Iba-1 intensities were quantified (Fig. 2b, lower panel). Moreover, microglia in intact non-Tg and GS-Tg brains did not significantly differ in their morphologies or densities (see 1 and 3 in Fig. 2a). These results suggest that GS-Tg microglia are less active in their response to brain injury.

Next, we compared the responses of non-Tg and GS-Tg microglia to laser-induced damage using two-photon-live imaging. To generate non-Tg and GS-Tg mice that expressed green fluorescent protein (GFP) in their microglia, we crossed GS-Tg heterozygous mice (GS/-) with Cx3cr1-GFP (GFP/GFP) mice, as previously described ${ }^{12}$. Upon laser injury, both non-Tg and GS-Tg microglia extended their processes towards damaged areas, isolating and covering them within $20 \mathrm{~min}$ (Fig. 2c). 
a

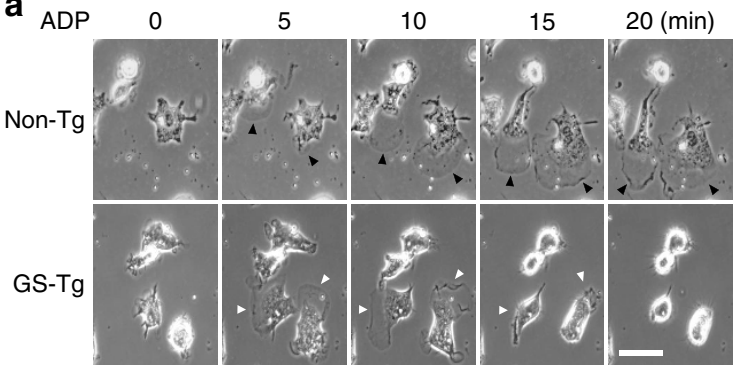

d
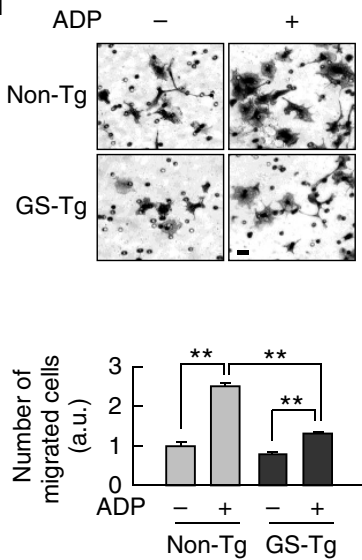

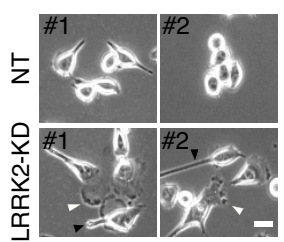

f

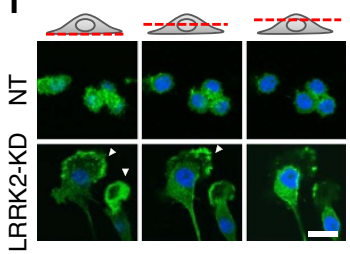

b
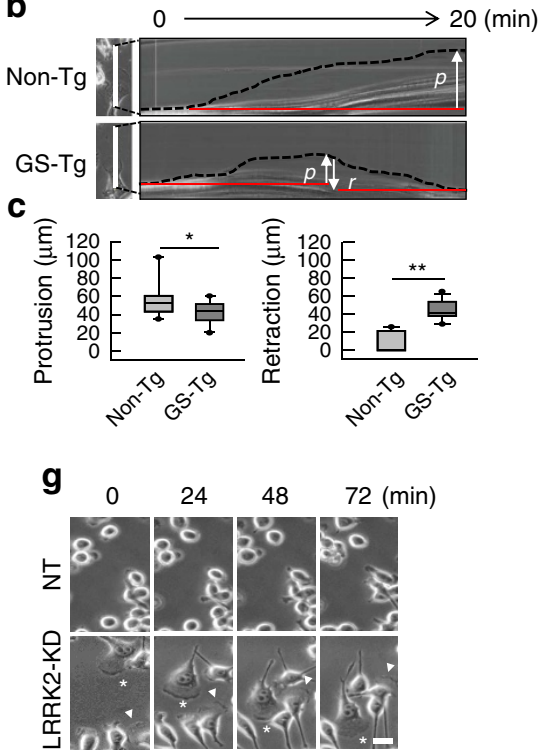

h

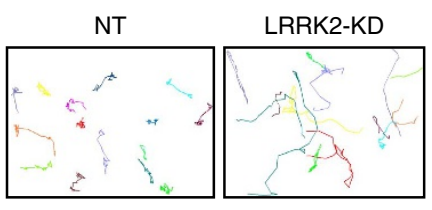

Figure 1 | Microglial motility is retarded and accelerated, respectively, by the LRRK2 GS mutation and LRRK2 deficiency. (a) Time-lapse images of non$\mathrm{Tg}$ and GS-Tg microglia were obtained every $5 \mathrm{~s}$ for $20 \mathrm{~min}$ after addition of $100 \mu \mathrm{M}$ ADP. Black and white arrowheads represent lamellipodia. (b,c) Membrane dynamics of microglia $(n=25)$ were quantified using SACED as described in the Methods section. Two-tailed Student's $t$-test, ${ }^{\star} P<0.05$, ${ }^{\star \star} P<0.01$. (d) Motilities of non-Tg and GS-Tg microglia were measured using transwells (upper panel). Number of cells migrated to the bottom of the transwells were counted at $12 \mathrm{~h}$ after plating (lower panel). Values are means \pm s.e.m. of three independent experiments. One-way ANOVA with NewmanKeuls post hoc test, ${ }^{\star \star} P<0.01$. (e) Unlike NT microglia, LRRK2-KD microglia showed a morphological polarity with a leading edge corresponding to lamellipodia (white arrowheads) and a tail (black arrowheads) even in the absence of ADP. (f) NT and LRRK2-KD cells were stained with Alexa-488 phalloidin, and Z-stack scanned images were obtained at 2- $\mu \mathrm{m}$ intervals from the bottom. Lamellipodia attached to the bottom were enriched with F-actin in LRRK2-KD cells (arrowheads). (g) Time-lapse images of NT and LRRK2-KD cells were taken at 2-min intervals for 72 min. Asterisks and arrow heads chased two different cells that moved in the images. (h) Spontaneous migration paths of NT and LRRK2-KD cells (15 cells each) were tracked for $6 \mathrm{~h}$. The location of each cell was determined every $10 \mathrm{~min}$ and connected to depict its migration route. Scale bar, $50 \mu \mathrm{m}(\mathbf{a}, \mathbf{d}) ; 10 \mu \mathrm{m}(\mathbf{e}-\mathbf{g})$. Data are representative of at least three independent experiments unless indicated. ANOVA, analysis of variance.

However, GS-Tg microglia showed a delayed tendency for wound isolation (Fig. 2c,d). Thus, our results collectively indicate that GS-Tg microglia are retarded in their ability to respond to brain injury, delaying the isolation of injured sites with respect to surrounding tissues.

LRRK2 interacts with FAK and inhibits its activation. Next, we examined how LRRK2 reduced microglial motility. Since LRRK2 is known to regulate actin dynamics ${ }^{5}$, we examined actin-related proteins that interact with LRRK2. In immunoprecipitation assays using mouse brain lysates, we detected LRRK2-Rac1 interactions, as previously reported ${ }^{2}$, but found little interaction of LRRK2 with other actin-related proteins, including paxillin, VASP (vasodilator-stimulated phosphoprotein), PAK (p21 protein-activated kinase 1) and cofilin (Supplementary Fig. 3). Interestingly, LRRK2 co-immunoprecipitated with FAK, a key regulator of cell migration, in mouse and human brain lysates and rat microglial lysates (Fig. 3a). We then determined which domains of FAK and LRRK2 were required for their interaction. HEK $293 \mathrm{~T}$ cells were transfected with full-length Myc-LRRK2 and FLAG-tagged domains of FAK, including
FERM, kinase and FAT domain, designated D1-D4 (Fig. 3b). In immunoprecipitation assays using anti-FLAG antibodies, LRRK2 bound to FERM and kinase domains of FAK but not to the FAT domain (Fig. 3b). We also found that kinase and WD40 domains of LRRK2 expressed in HEK 293T cells interacted with FAK (Fig. 3c). Therefore, these results suggest that LRRK2 and FAK directly bind each other through kinase and WD40 domains of LRRK2 and FERM and kinase domains of FAK.

Next, we examined whether LRRK2 regulates microglial movement through FAK activation. We first examined whether FAK mediates ADP-induced microglial motility. In rat microglia, ADP treatment rapidly (within $1-5 \mathrm{~min}$ ) induced FAK activation, which was monitored based on the autophosphorylation of FAK $(\mathrm{pY} 397)^{37}$. Thereafter, the levels of pY397 decreased to the baseline at about $30 \mathrm{~min}$ (Supplementary Fig. 4a). Then, we treated two specific FAK inhibitors, PF573228 and FAK inhibitor 14 on microglia. These inhibitors have been known to be 50-250-fold more selective for FAK than for other kinases including Pyk2, CDK1/7, GSK-3 $\beta$ and receptor tyrosine kinases, EGFR, PDGFR and IGF-RI ${ }^{38,39}$. In the presence of inhibitors, these cells failed to form stable lamellipodia (Supplementary Fig. 4b) or showed decreased movement in the presence of ADP; 
a
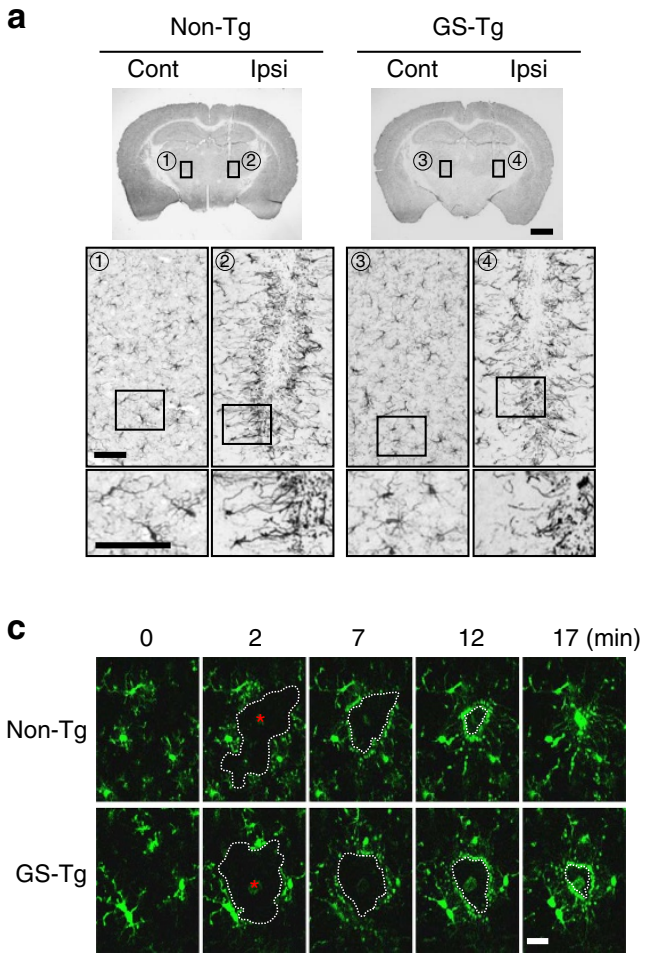

b
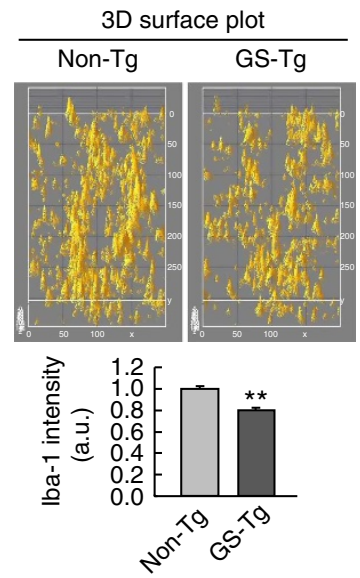

d

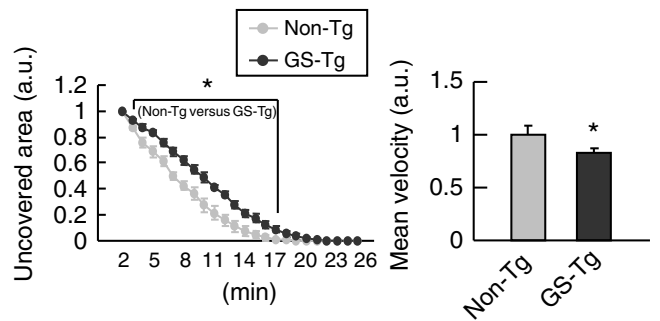

Figure 2 | GS-Tg microglia slowly and inefficiently respond to brain injury. (a) Stab-wound injuries were produced in the striatum of non-Tg and GS-Tg mice, and microglia were analysed with lba-1 antibodies at $1 \mathrm{~h}$ post injury. The most highly damaged sections were photographed. The middle and lower panels show higher magnifications of the areas boxed in the upper and middle panels, respectively. The contralateral sides (cont) were used as uninjured controls. (b) On the basis of the lba-1 intensities obtained from the results shown in the middle panels of a, 3D surface plots were obtained and quantified using Image J software. Values are given as the means \pm s.e.m. of four animals. (c) GS-Tg microglia respond more slowly to laser-induced injury compared with non-Tg microglia. Laser injury was applied at 2 min to the cortices of 3-month-old non-Tg and GS-Tg mice expressing GFP in their microglia, and the behaviour of microglia was monitored by time-lapse imaging for at least $25 \mathrm{~min}$. Asterisks: the sites laser injury was applied. (d) For measurement of the microglial response to injury, Image Analysis Software (Perkin-Elmer) was used to analyse the areas that were not covered by microglia (left panel) and the velocity of microglial processes moved towards the injury sites (right panel). Values are given as the means \pm s.e.m. of four animals. Two-tailed Student's $t$-test, ${ }^{\star} P<0.05 ;{ }^{\star} P<0.01$. Scale bars, $1 \mathrm{~mm}$ (a, upper panel); $100 \mu \mathrm{m}$ (a, middle and lower panels); $20 \mu \mathrm{m}$ (c).

in this, they were similar to GS-Tg microglia (Supplementary Fig. $4 \mathrm{~b}-\mathrm{e}$ versus Fig. 1a-d). Next, we found that pY397-FAK levels were higher in LRRK2-KD BV2 cells than in NT BV2 cells (Fig. 4a). Furthermore, ADP increased pY397-FAK levels to a lesser extent in GS-Tg microglia than in non- $\mathrm{Tg}$ microglia although basal pY397-FAK levels were little different (Fig. 4b). Interestingly, pY397-FAK levels were lower in midbrain lysates prepared from 8-week-old GS-Tg mice than in lysates prepared from age-matched, littermate control non-Tg mice (Fig. 4c). We further examined the effect of LRRK2 on Y397 phosphorylation by measuring pY397-FAK levels in HEK 293T cells in response to ADP, with or without transfection of various LRRK2 expression constructs. In untransfected HEK 293T cells, ADP induced an increase in pY397-FAK levels that reached a peaked at about $5 \mathrm{~min}$ (Fig. 4d). As was the case in microglial cells and brain lysates, the pathogenic GS-LRRK2 mutant significantly reduced pY397-FAK levels compared with WT-LRRK2 and the kinase dead mutant, D1994A (DA)-LRRK2, in response to ADP treatment (Fig. 4e). These results strongly suggest that LRRK2 regulates microglial motility through inhibition of FAK activation.

LRRK2 phosphorylates TXR motifs in FAK.. The next question arising was how LRRK2, a Ser/Thr kinase ${ }^{1}$, regulates phosphorylation of Y397-FAK. In vitro kinase assays using WT and mutant versions of recombinant LRRK2 proteins, and
GST-FAK proteins showed phosphorylation of FAK in the absence of LRRK2 (Fig. 5a). In the presence of WT-LRRK2 or GS-LRRK2, the phosphorylation levels of FAK further increased, with GS-LRRK2 producing a greater effect (Fig. 5a). In contrast, DA-LRRK2 did not increase FAK phosphorylation beyond basal phosphorylation level (Fig. 5a). We further examined whether LRRK2 increased the phosphorylation capacity of FAK. In vitro kinase assay using K454R-FAK (KR-FAK), a kinase-dead FAK mutant ${ }^{40}$, revealed that GS-LRRK2 induced a concentrationdependent increase in the phosphorylation level of KR-FAK, whereas KR-FAK alone was barely phosphorylated (Fig. 5b). These results demonstrate that LRRK2 directly phosphorylates FAK, indicating that FAK is a substrate of LRRK2 kinase.

It has been reported that LRRK2 favours phosphorylation of threonine residues in the $\operatorname{TXR}(\mathrm{K})$ motif, a consensus target sequence of LRRK2 (refs 41,42). We identified six TXR(K) consensus sequences in FAK (Fig. $5 \mathrm{c}$ ), and examined whether LRRK2 phosphorylates the TXR $(\mathrm{K})$ motif(s) by performing in vitro kinase assays using non-radiolabelled cold ATP followed by western analyses using pTXR-specific antibody (Fig. 5d). As expected, LRRK2 increased pTXR-FAK levels, exhibiting a rank order of effectiveness of GS $>$ WT $>$ DA (Fig. 5d). We next used site-directed mutagenesis to introduce a phospho-mimetic glutamate (Glu, E) residue in place of the Thr residue in each TXR(K) motif of FLAG-tagged FAK to mimic LRRK2-induced phosphorylation. After expressing the mutant constructs in HEK $293 \mathrm{~T}$ cells, we immunoprecipitated FAK with FLAG antibodies and 
a

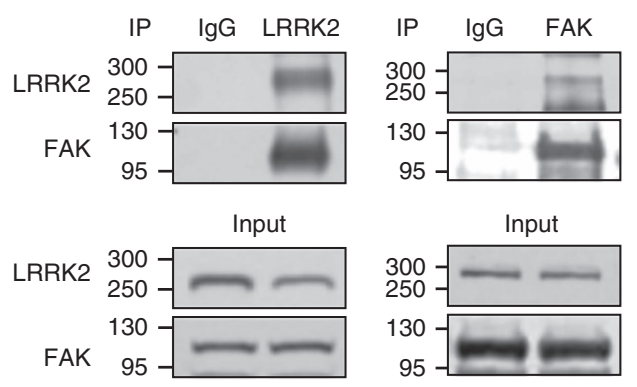

Mouse brain

b
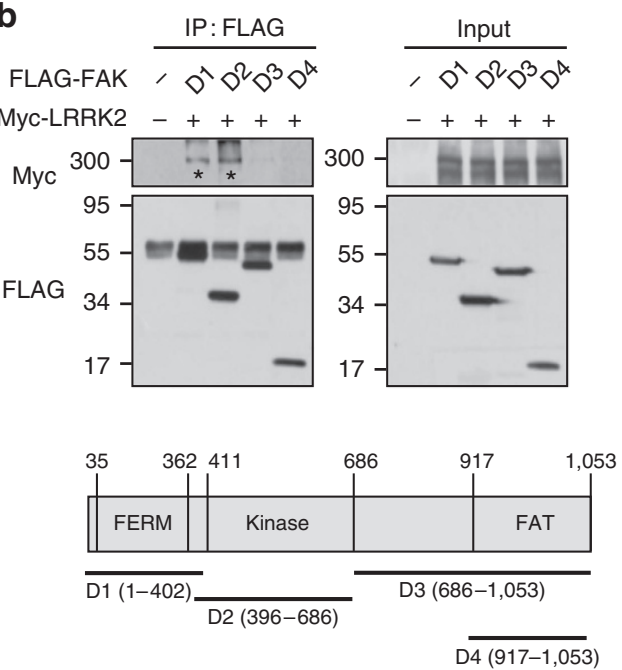
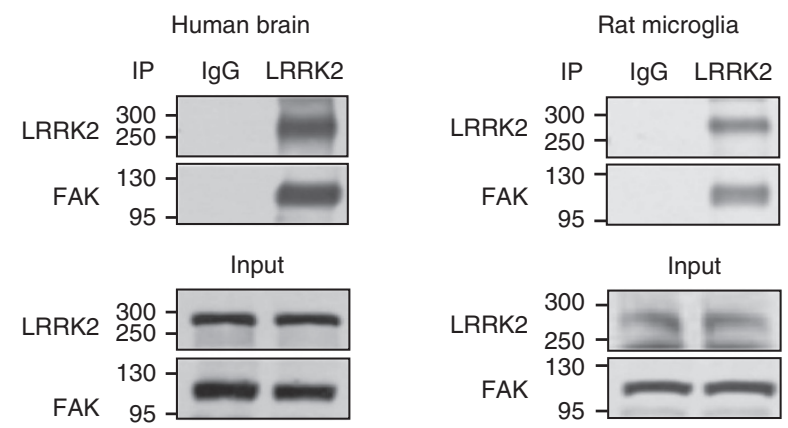

C

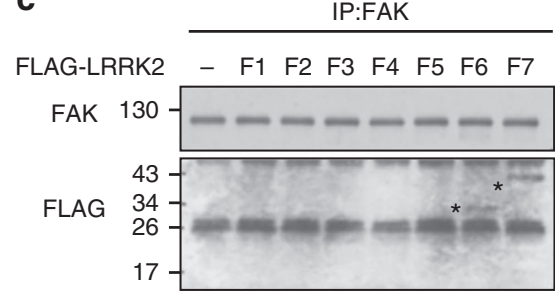

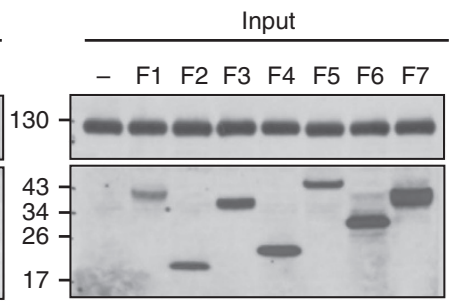

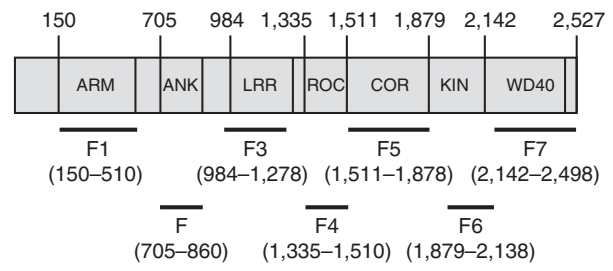

Figure 3 | LRRK2 interacts with FAK. (a) Lysates were prepared from mouse and human brain, or rat primary microglia and immunoprecipitated (IP) using antibodies specific for LRRK2 and FAK. Immunoprecipitated proteins were analysed by western blot using antibodies specific for LRRK2 and FAK. IgG was used as a negative control. Levels of each protein in lysates were analysed using the indicated antibodies (input). (b,c) HEK 293T cells were transfected with Myc-tagged LRRK2 and FLAG-tagged FAK domains (D1-D4, lower panel) (b) or FLAG-tagged LRRK2 domains (F1-F7, lower panel) (c). Cell lysates were prepared $48 \mathrm{~h}$ after transfection, and immunoprecipitation was carried out using anti-FLAG (b) or anti-FAK (c) antibodies; proteins in IP complexes were identified by western blotting. Inputs in $\mathbf{b}$ and $\mathbf{c}$ show the amount of each protein used for IP. * indicates LRRK2 (b) or LRRK2 domains (c) that interacted with FAK. Data are representative of at least three independent experiments.

measured the pY397-FAK levels of each mutant. Interestingly, replacement of T474 with Glu (T474E) strongly suppressed FAK Y397 phosphorylation (Fig. 5e). However, replacement of Thr residues in other TXR motifs had no effect on FAK Y397 phosphorylation (Fig. 5e). We further examined whether LRRK2 directly phosphorylated FAK T474 by comparing TXR phosphorylation levels of WT-FAK and T474A-FAK. To this end, we expressed FLAG-tagged WT-FAK and T474A-FAK in HEK 293T cells, immunoprecipitated them with FLAG antibody, and then carried out in vitro kinase assays using recombinant GS-LRRK2 protein (Fig. 5f). We also co-transfected HEK 293T cells with FLAG-tagged WT-FAK and T474A-FAK with GS-LRRK2. pTXR levels of FAK were analysed after immunoprecipitation using FALG antibody followed by western blot using pTXR antibody (Fig. 5g). GS-LRRK2 enhanced pTXR level of WT-FAK but not that of T474A-FAK in both conditions (Fig. 5f,g). Taken together, these results suggest that LRRK2 directly phosphorylate FAK at T474.

LRRK2 interacts with FAK in the cytoplasm. Next, we examined where the two proteins interacted using two LRRK2-specific antibodies, N231B/34 (Fig. 6) and N241A/34 (Supplementary Fig. 5) that did not produce LRRK2 signal in LRRK2 knockout neurons ${ }^{43}$. In non-Tg and GS-Tg microglia cultured, LRRK2 was found in the cytoplasm near the nucleus in the absence of ADP (see 1 and 10 in Fig. 6a and 1 and 10 in Supplementary Fig. 5a), while FAK was located in both the cytoplasm and the nucleus (see 2, 3, 11 and 12 in Fig. 6a and 2, 3, 11 and 12 in Supplementary Fig. 5a). Following ADP treatment, FAK was obviously detectable in the lamellipodia (see 5, 6, 14 and 15 in Fig. 6a and 5, 6, 14 and 15 in Supplementary Fig. 5a) while most of LRRK2 remained in the cytoplasm near the nucleus (see 4, 6, 13 and 15 in Fig. 6a and 4, 6, 13 and 15 in Supplementary Fig. 5a). In magnified figures, a small portion of LRRK2 was found in the lamellipodia (see arrows present in 7-9 and 16-18 in Fig. 6a and 7-9 and 16-18 Supplementary Fig. 5a), but not at the edge of it where FAK was abundantly detectable (see arrowheads present in 7-9 and 16-18 in Fig. 6a and 7-9 and 16-18 Supplementary Fig. 5a). In addition, LRRK2 was not co-localized with FAK in the lamellipodia (see 9 and 18 in Fig. $6 a$ and 9 and 18 in Supplementary Fig. 5a). Using a proximity ligation assay (PLA), we further examined interaction between LRRK2 and FAK. As shown in immunostaining (Fig. 6a), PLA spots were not detectable in the leading edge (Fig. 6b; Supplementary Fig. 5b), suggesting the interaction between two proteins mainly occurred in the cytoplasm. In addition, a portion of LRRK2 regardless of WT and GS appeared to consistently interact with FAK since the amounts of spots were not changed by ADP treatment and GS mutation (Fig. 6b; Supplementary Fig. 5b,c). Furthermore, locations of spots were not limited to the perinuclear region 
a BV2 cell

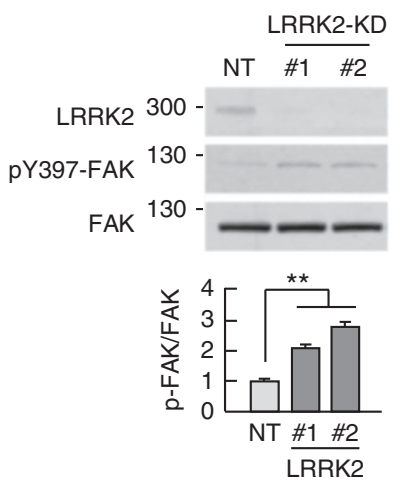

b Primary microglia

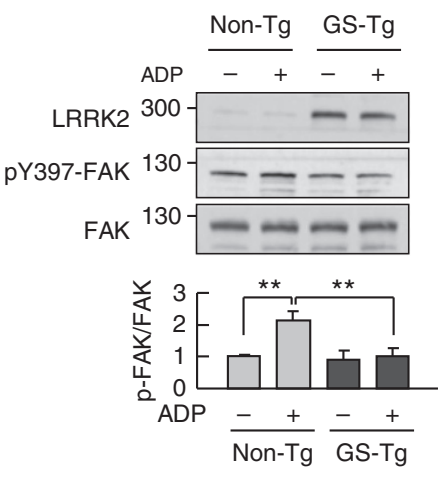

C

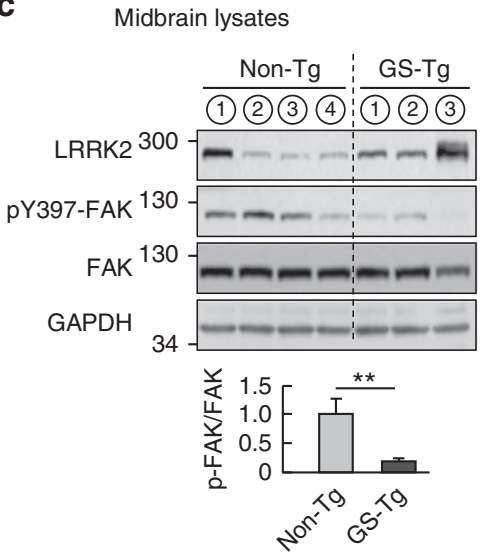

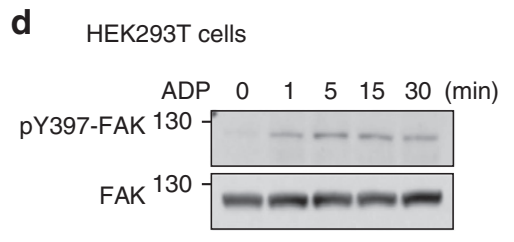

e

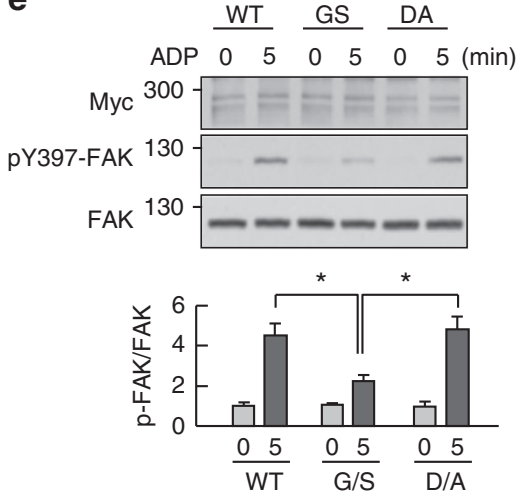

Figure 4 | LRRK2 negatively regulates FAK activation. (a) The effect of LRRK2 on FAK activation was analysed by western blot using antibodies specific for pY397-FAK, FAK and LRRK2 (upper panels) in LRRK2-KD cells (\#1 and \#2) and a control (NT) cells used in Fig. 1. Band intensities of pY397-FAK were quantified, normalized to that of FAK and plotted (lower panels). Values are means \pm s.e.m. of three separate experiments. (b) Microglia cultured from non-Tg or GS-Tg mouse brains were treated with $100 \mu \mathrm{M}$ ADP for $5 \mathrm{~min}$, and the levels of pY397-FAK, FAK and LRRK2 were analysed. Values are means \pm s.e.m. of five separate experiments. (c) Midbrain lysates were prepared from 8-week-old GS-Tg mice and littermate non-Tg mice. Each number indicates a different animal. Values are means \pm s.e.m. of three or four animals. (d) HEK 293T cells were treated with ADP (100 $\mu$ M) for the indicated times, and the levels of pY397-FAK were measured. (e) HEK 293T cells were transfected with Myc-tagged WT-LRRK2, GS-LRRK2 and DA-LRRK2 mutants. Forty-eight hours later, cells were treated with $\operatorname{ADP}(100 \mu \mathrm{M})$ for the indicated times. Data are representative of three independent experiments. Values are means \pm s.e.m. of three independent experiments. One-way ANOVA with Newman-Keuls post hoc test, ${ }^{\star} P<0.05,{ }^{\star \star} P<0.01$ in $\mathbf{a}, \mathbf{b}$ and $\mathbf{e}$. Two-tailed Student's $t$-test, ${ }^{\star \star} P<0.01$ in c. ANOVA, analysis of variance.

where LRRK2 was densely located, spots were also found in the region where LRRK2 was sparsely located (Fig. 6b; Supplementary Fig. 5b), indicating that the amount of LRRK2 minimally affected interaction with FAK. In control experiments with single primary antibodies or without any antibodies, PLA spots were barely detectable (Supplementary Fig. 5b,c).

Using HEK 293T cells, we further analysed whether the LRRK2 mutation altered the interaction between FAK and LRRK2. The interaction was detectable in the cytoplasm in the absence or presence of ADP (Supplementary Fig. 6a); the amount of FAK that co-immunoprecipitated with LRRK2 was not changed by the absence/presence of ADP regardless of FAK activation (as demonstrated by Y397 phosphorylation; Fig. 7a). Furthermore, similar amounts of LRRK2 WT, GS mutant and DA mutant coimmunoprecipitated with FAK (Fig. 7b). Next, we examined whether these LRRK2 mutations affected the formation of pY397-FAK-positive focal adhesions in non-Tg, GS-Tg microglia and HEK 293 T cells (Fig. 7c,d). ADP treatment rapidly (within $5 \mathrm{~min}$ ) increased pY397-FAK-positive focal adhesion in the lamellipodia in non-Tg microglia, but not in GS-Tg microglia (Fig. 7c). In HEK $293 \mathrm{~T}$ cells, following ADP treatment, pY397-FAK-positive focal adhesions were formed at the cell edges within $5 \mathrm{~min}$. These adhesions were maintained at $30 \mathrm{~min}$ but disappeared by $180 \mathrm{~min}$ in GFP-mock (GFP)-, WT-LRRK2 (WT)- and DA-LRRK2 (DA)-transfected cells (Fig. 7d). Similar with GS-Tg microglia, however, GS-LRRK2 (GS)-transfected cells showed significantly fewer pY397-FAK-positive focal adhesions (Fig. 7d). Taken together, these results indicate that LRRK2 regulates the cytoplasmic activation of FAK, and suggest that the enhanced inhibitory effect of the LRRK2 G2019S mutant on FAK activation may not be due to an increased interaction between the two proteins.

Effect of LRRK2 inhibitor on FAK and microglial motility. Next, we examined whether inhibition of LRRK2 kinase activity rescued pY397-FAK levels using GSK2578215A (GSK), a LRRK2 inhibitor ${ }^{44}$. In GS-LRRK2-expressing HEK 293T cells, phosphorylation of LRRK2 at S935 (pS935) was decreased with GSK about $60 \%$, confirming that this inhibitor effectively reduced LRRK2 kinase activity, as previously reported ${ }^{44}$ (Fig. 8a). We initially chose two well-known LRRK2 kinase inhibitors, IN-1 (ref. 45) and CZC 54254 (ref. 46). However, IN-1 and CZC 54254 have off-target effects on FAK ${ }^{45,46}$, whereas GSK has no such effect ${ }^{44}$. In our in vitro kinase assay also showed that 


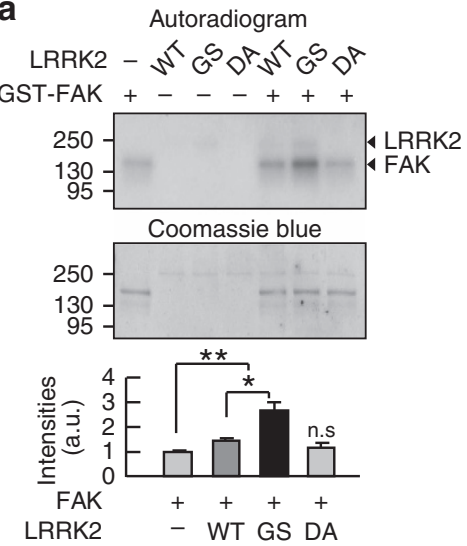

d LRRK2 - WT GS DA
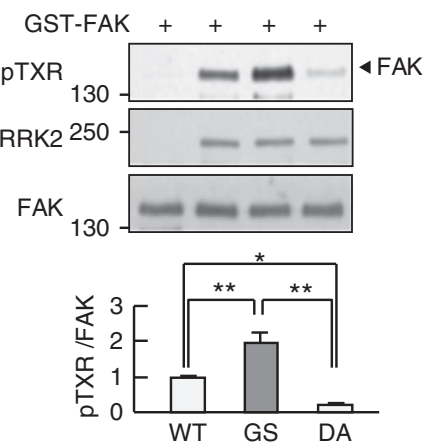

b

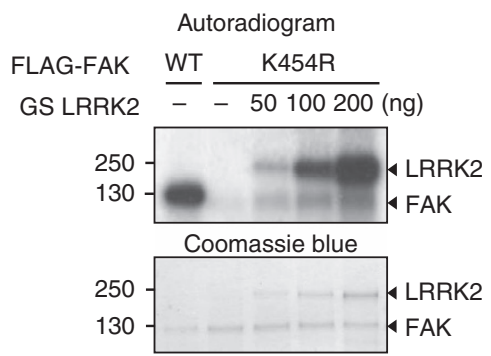

C LRRK2 kinase consensus sites

...211 LLDSVKAKTLRKLIQQTFRG...

284

281 SYLTDKGCNPTHLADFTG. .

455

451 VAIKTCKNCTSDSVREKFLQ..

474

471 EALTMROFDH...

979

..971 TIPLLPASTHREIEMAGKLL. . e

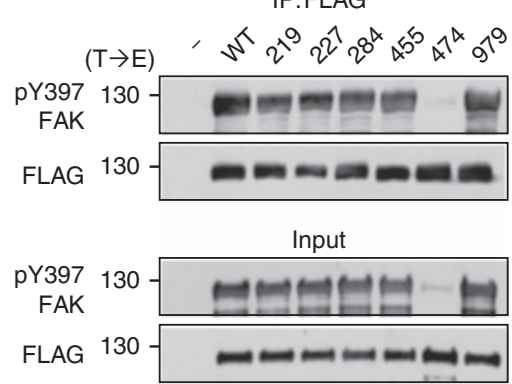

f

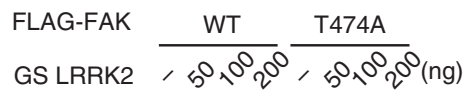
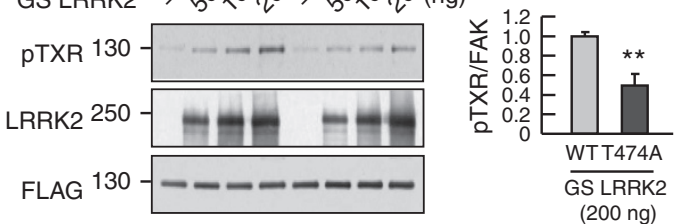

g

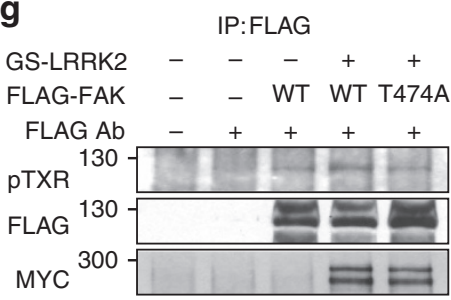

Figure 5 | LRRK2 suppresses FAK Y397 phosphorylation through phosphorylation of TXR motif(s) in FAK. (a,b,d,f) In vitro kinase assays were carried out using 250 ng recombinant proteins of GST-FAK (a,d) FLAG-tagged WT, K454R mutant FAK (b) or T474A mutant FAK (f) and WT-, GS- and DA-LRRK2 recombinant proteins ( $50 \mathrm{ng}$ in $\mathbf{a}, \mathbf{d}$ or $50-200 \mathrm{ng}$ in $\mathbf{b}$ and $\mathbf{f}$ ) as indicated. ${ }^{32}$ P-labelled FAK was detected in autoradiograms (upper panels in $\mathbf{a}$ and $\mathbf{b}$ ). Coomassie blue staining shows the amount of proteins in each reaction mixture (lower panels in $\mathbf{a}$ and $\mathbf{b}$ ). In $\mathbf{d}$ and $\mathbf{f}$, in vitro kinase assays were carried out using cold ATP without ${ }^{32}$ P-ATP, and the reaction mixtures were analysed by western blot using antibodies specific for FLAG, pTXR, FAK and LRRK2. Values are means \pm s.e.m. of three separate experiments. Data are representative of three independent experiments. (c) TXR $(K)$ phosphorylation motifs found in FAK are indicated in bold. $(\mathbf{e}, \mathbf{g})$ Thr residues in six TXR sites (each number indicates the amino acid number in $\mathbf{c}$ ) were mutated to Glu (T $\rightarrow E$ )

(e). HEK 293T cells were transfected with FLAG-tagged WT-FAK or six $(T \rightarrow E)$ FAK mutants (e) or FLAG-tagged WT-FAK or T474A mutant FAK with GSLRRK2 (g) for $48 \mathrm{~h}$. Whole-cell lysates were prepared and immunoprecipitated with anti-FLAG antibody, and the levels of pY397-FAK and FLAG (e) or PTXR, Myc and FLAG (g) were analysed by western blot. One-way ANOVA with Newman-Keuls post hoc test in $\mathbf{a}$ and $\mathbf{d}{ }^{\star} P<0.05$, ${ }^{\star \star} P<0.01$. Two-tailed Student's $t$-test in $\mathbf{f}$. ${ }^{\star} P<0.01$. ANOVA, analysis of variance.

IN-1 and CZC 54254 strongly suppressed basal phosphorylation of FAK in a dose-dependent manner (Supplementary Fig. 7a). Accordingly, these inhibitors did not rescue pY397-FAK in GS-LRRK2-expressing cells (Supplementary Fig. 7b). On the other hand, GSK induced increase in pY397-FAK levels while decreasing pS935 levels (Fig. 8a). In vitro kinase assays also showed that GSK eliminated the increase in pTXR-FAK levels induced by GS-LRRK2, reducing them to the levels of DA-LRRK2 or GST-FAK only (Fig. 8b). Moreover, GSK also changed microglial responses to ADP, markedly increasing the formation of stable lamellipodia in GS-Tg microglia (arrowheads in Fig. 8c and Supplementary Movie 3). SACED analysis further showed that GSK significantly increased protrusion but not affected retraction (Fig. 8e). Similar to GSK, GW5074, another inhibitor that potentially inhibits LRRK2 kinase activity ${ }^{47}$, inhibited the effects of GS-LRRK2: it increased pY397-FAK levels in GS-LRRK2-overexpressing HEK 293T cells, decreased pTXR levels produced by GS-LRRK2 in vitro kinase assay, rescued defect of stable lamellipodia formation, and increased cell migration (Supplementary Fig. 8; Supplementary Movie 4). Although GW5074 has been developed to inhibit Raf-1 kinase $^{48}$, it is hard to think the effect of GW5074 on microglial motility and FAK is due to Raf- 1 inhibition since Raf- 1 inhibition rather retards cell motility ${ }^{49}$. Taken together, these results suggest that LRRK2 phosphorylates TXR motif(s) in FAK, which inhibits microglial motility through inhibition of Y397 phosphorylation. On the basis of this, we propose that the LRRK2 kinase inhibitors could potentially be used to treat and/or prevent PD by restoring the motility of G2019S microglia.

\section{Discussion}

We herein report that microglia carrying the LRRK2 G2019S mutation (GS-Tg microglia) show retarded motility and fail to isolate injury sites as quickly and efficiently as non-Tg microglia. On the basis of results in this study, we proposed a model how 

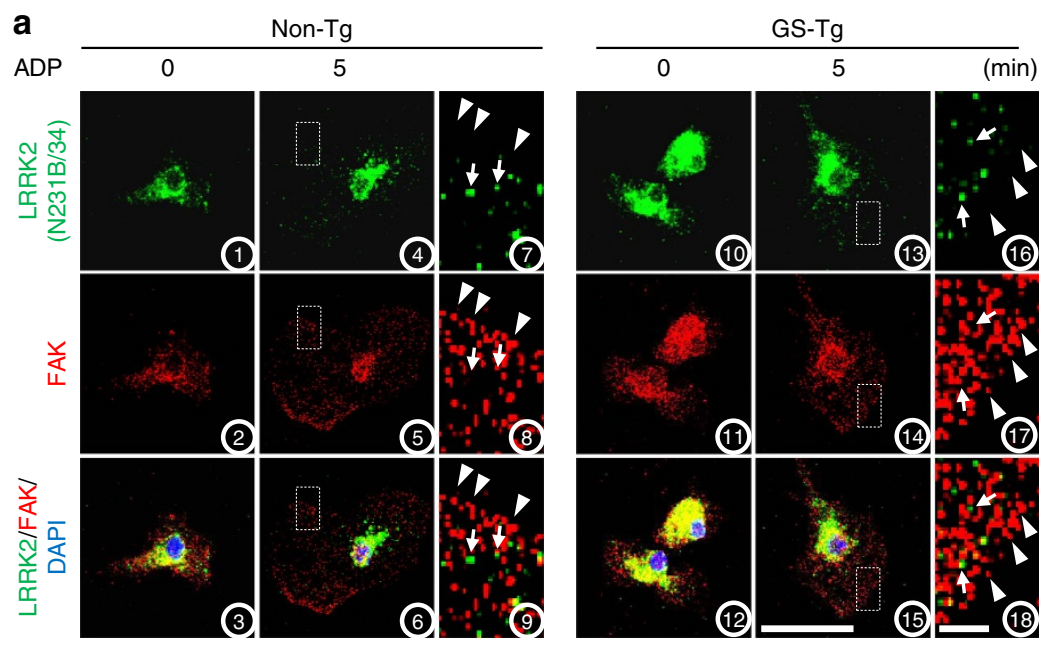

b

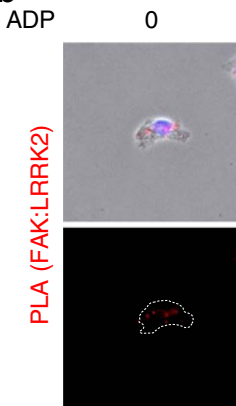

5
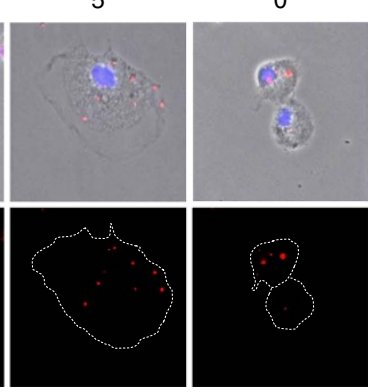

5
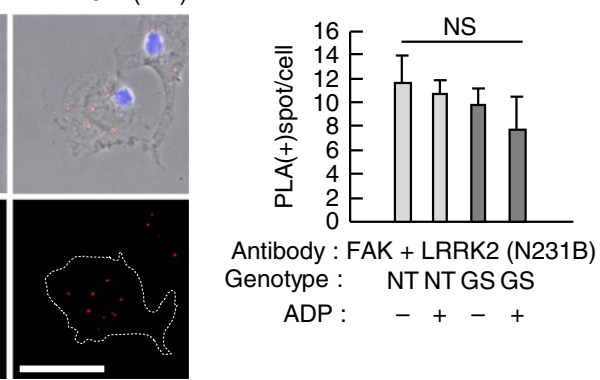

Figure 6 | LRRK2 interacts with FAK in the cytoplasm. (a,b) Non-Tg and GS-Tg microglia were treated with $100 \mu$ M ADP for 5 min. (a) Cells were stained with specific antibodies against FAK and LRRK2 (N231B/34). Magnified images of boxed areas in 4-6 and 13-15 were shown in 7-9 and 16-18, respectively. Arrows and arrowheads indicate location of LRRK2 and FAK, respectively. (b) The interaction between FAK and LRRK2 was analysed in non-Tg and GS-Tg microglia $(n=50)$ using in situ PLA, as described in the Methods section. White dotted lines indicate the cell boundaries (b, lower panel). PLA spots (representing interactions between the two proteins) were quantified (b, right panel). Values are means \pm s.e.m. of three separate experiments. NS, not significant. Scale bars, $50 \mu \mathrm{m}(\mathbf{a}, 15 ; \mathbf{b}) ; 5 \mu \mathrm{m}(\mathbf{a}, 18)$.

LRRK2 and GS-LRRK2 regulate microglial motility (Fig. 9): when ADP binds to $\mathrm{P}_{2} \mathrm{Y}_{12}$ receptors, FAK is activated (marked by pY397-FAK) through PLC $\beta$-, and $\mathrm{Ca}^{2+}$-dependent pathways (Supplementary Fig. 9), which is necessary for stable lamellipodia formation and proper microglial movement. In this process, LRRK2 negatively regulates microglial movement through direct interaction and phosphorylation of FAK on T474 in TXR motif, which prevents phosphorylation of FAK Y397. Mechanistically, G2019S-LRRK2 mutation having increased kinase activity retards microglial motility via the excessive inhibition of FAK activation (Fig. 9). Since the observed defect in the ability of these microglia to isolate injury sites will lead to worsening of damage ${ }^{15}$, our results collectively suggest that microglial defects caused by the G2019S mutation may contribute to the development of PD.

Since LRRK2 interacts with actin, and the actin-regulatory proteins $^{3,4,10}$, we hypothesized that it could regulate microglial motility. Indeed, our results revealed that LRRK2 regulated microglial motility (Figs 1,2). LRRK2-KD BV2 microglia were morphologically different from NT microglia, and highly motile even in the absence of any stimulators (Fig. 1e-h). Although GS-Tg microglia were not different from non-Tg microglia in morphology in culture and in intact brain (Figs 1a and 2a), these cells showed defects in response to ADP (Fig. 1a-c; Supplementary Fig. 1), or brain injury (Fig. 2). This suggests that the ability of LRRK2 to regulate microglial motility is related to its kinase activity. However, a recent study reported that GS-LRRK2 enhanced chemotactic responses of macrophages ${ }^{50}$. Fibroblasts derived from GS PD patients and R1441G mice also increased motility compared with those cells from non-PD patients and WT mice, respectively, through direct interaction with tubulin ${ }^{51}$. For microglial movement, actin dynamics is important ${ }^{15}$. Previously, it has been reported that actin and microtubule differently regulate movement of different types of cells ${ }^{52}$. Therefore, LRRK2 may differently regulate motility of cells depending on cell types and microenvironment of tissues.

We identified FAK as a substrate of LRRK2, demonstrating that FAK interacted with LRRK2 in cultured rat microglia, as well as mouse and human brain lysates (Fig. 3a). LRRK2 bound through its kinase and WD40 domains to FAK FERM and kinase domains (Fig. 3b,c). FAK has long been known as a critical player in processes that regulate cell movement ${ }^{26,53}$. In response to stimuli that induce motility of cells, FAK becomes autophosphorylated on Y397 (refs 54-57), which is essential for lamellipodial formation and progression of migrating cells ${ }^{58}$. FAK is also phosphorylated on Y576/577, S732, Y861, Y863 and Y925 (refs 54-57,59). All of these phosphorylations enhance FAK activity and cell migration ${ }^{54-57,59}$. In this study, we provide a phosphorylation-induced negative regulatory mechanism of FAK. LRRK2-KD microglia showed higher levels of pY397 even in the absence of any stimulators (Fig. 4a). Conversely, microglia from Tg mice expressing the gainof-function G2019S-LRRK2 mutant showed decreased pY397-FAK levels (Fig. 4b). This seemingly incongruous finding that the Ser/Thr kinase activity of LRRK2 is responsible for inhibiting FAK activation (Fig. 4) suggests that a complicated mechanism is involved in 
a

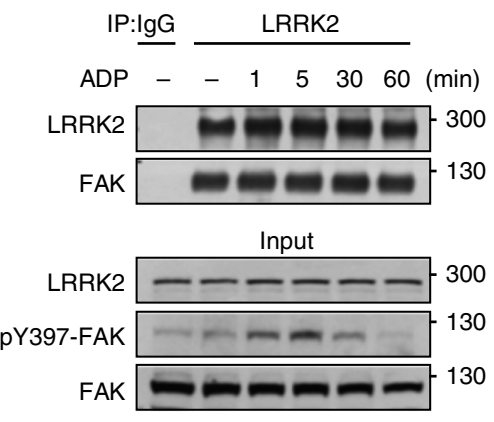

b

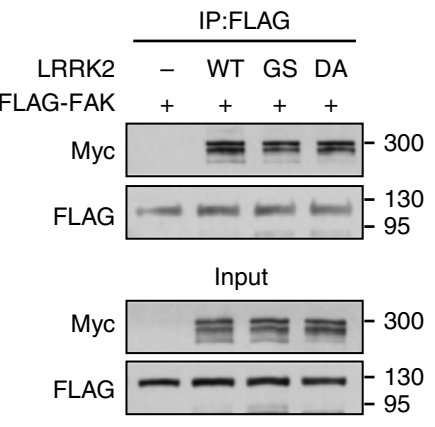

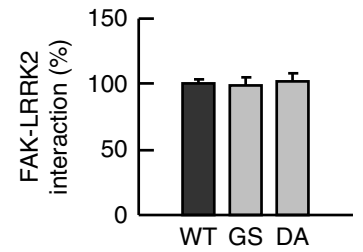

C

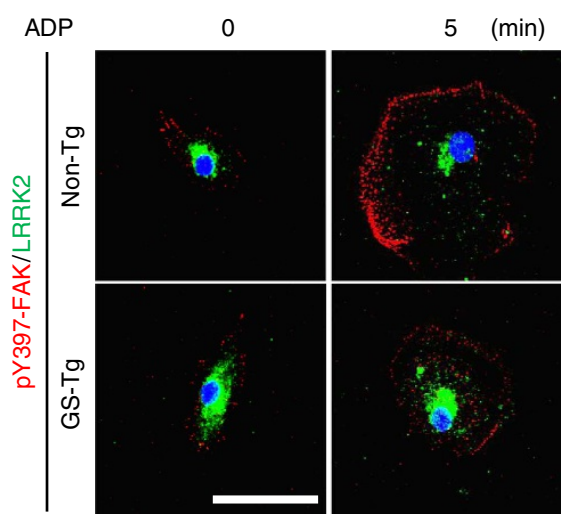

d
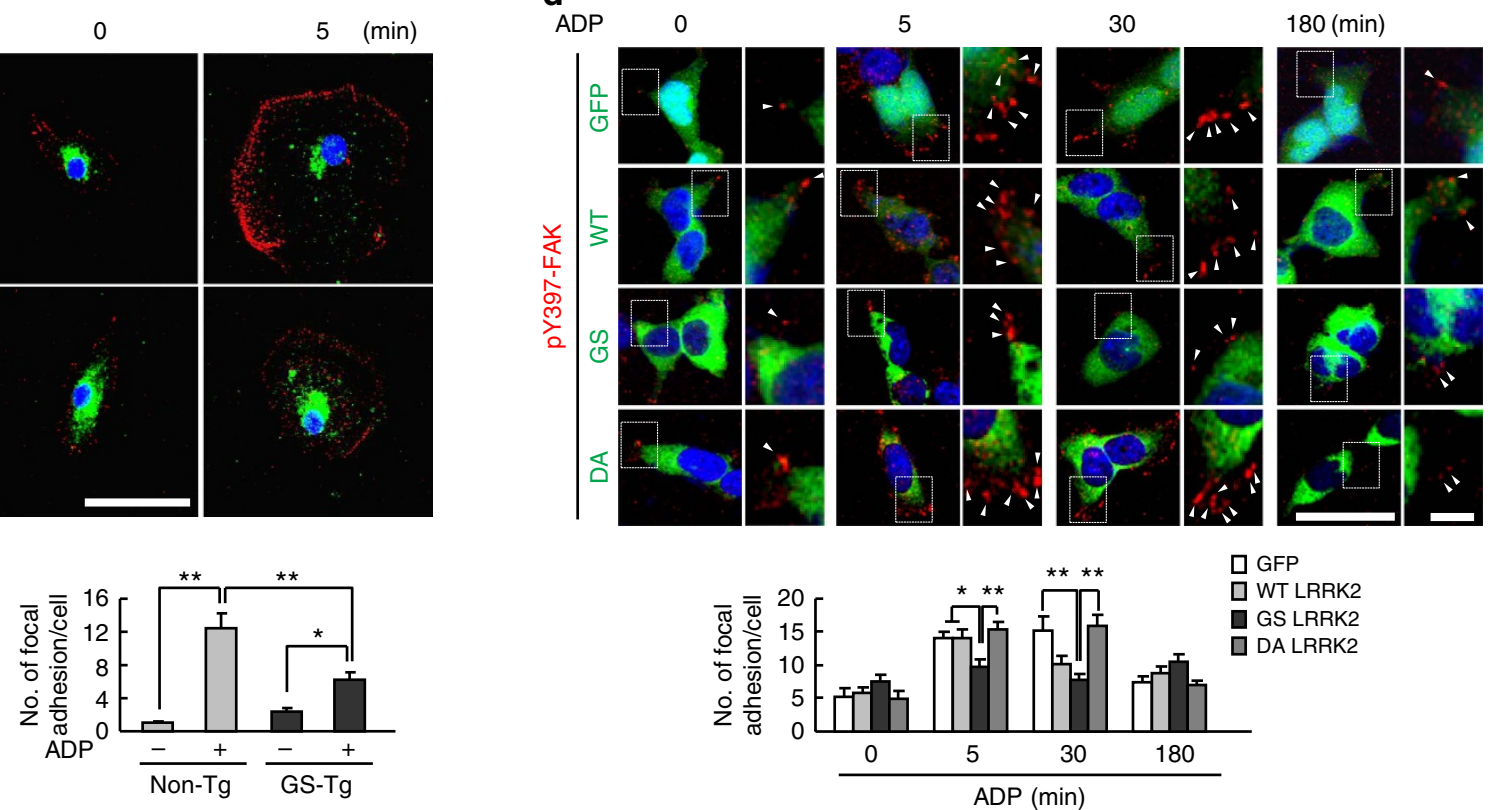

Figure 7 | Effect of ADP and LRRK2 mutation on the LRRK2-FAK interaction and focal adhesion formation. (a) ADP does not affect the interaction between LRRK2 and FAK. HEK 293T cells were treated with $100 \mu \mathrm{M}$ ADP for the indicated times, harvested and immunoprecipitated with an antibody against LRRK2. The amounts of FAK that co-immunoprecipitated with LRRK2 were analysed by western blot using antibodies specific for LRRK2 and FAK (left panel), and the results were quantified (right panel). IgG was used as a negative control. The levels of LRRK2 and pY397-FAK in lysates were analysed by western blot (input). (b) LRRK2 mutation does not affect the LRRK2-FAK interaction. HEK 293T cells were transfected with vectors encoding FLAG-FAK and Myc-LRRK2 (WT, GS or DA). Immunoprecipitation was performed using FLAG antibodies, the amounts of immunoprecipitated LRRK2 were analysed with western blot, and the results were quantified. (c,d) Non-Tg and GS-Tg microglia $(n=25)(\mathbf{c})$ and HEK 293T cells transfected with Myc-LRRK2 (WT, GS or DA) vectors $(n=50)$ (d) were treated with $100 \mu \mathrm{M}$ ADP for the indicated times, fixed and stained with antibodies against pY397-FAK and LRRK2, and pY397-FAK and GFP or Myc (WT, GS and DA), respectively. The pY397-FAK-positive focal adhesions were counted using the Image J software (lower panel). ${ }^{\star} P<0.05$ and ${ }^{\star} P<0.01$ by one-way ANOVA with the Newman-Keuls post hoc test. Scale bar, $50 \mu m(\mathbf{b})$. Data are representative of at least three independent experiments. ANOVA, analysis of variance.

LRRK2-mediated inhibition of FAK Y397 phosphorylation. It has been reported that LRRK2 favours phosphorylation of Thr residue(s) in $\operatorname{TXR}(\mathrm{K})$ motifs $^{41,42}$; six such sites are present in FAK (Fig. 5c). In a series of experiments, we found that LRRK2 phosphorylated at least one Thr residues in FAK TXR(K) motifs, namely Thr474, which in turn resulted in the inhibition of Y397 autophosphorylation. Replacing T474 in the FAK kinase domain with a phospho-mimic glutamate (T474E) completely eliminated FAK Y397 autophosphorylation (Fig. 5e); moreover, introduction of a non-phosphorylatable alanine at this same site (T474A) significantly decreased pTXR levels compared with that observed in WT-FAK in the presence of G2019S-LRRK2 (Fig. 5f,g), suggesting that T474 site is a LRRK2 target. Accordingly, a LRRK2 kinase inhibitor, GSK2578215A, increased pY397-FAK levels and reversed the motility phenotype of GS-Tg microglia (Fig. 8; Supplementary Movie 3). Therefore, LRRK2 appeared to decrease FAK Y397 phosphorylation through direct phosphorylation of at least one TXR/K motif (T474). However, we do not exclude possibilities that LRRK2 inhibits FAK Y397 phosphorylation through other mechanisms. Although kinase-dead DA-LRRK2 (ref. 60) did not increase pTXR to the same extent as WT-LRRK2 in in vitro kinase assays (Fig. 5d), pY397-FAK levels were not different in DA- and WT-LRRK2-expressing cells (Fig. 4e). Therefore, LRRK2 may decrease FAK Y397 phosphorylation through direct and/or indirect interaction with other signalling molecules that inhibit FAK Y397 phosphorylation such as phosphatases and/or suppressor of cytokine signalling proteins $s^{61-64}$.

The interaction between LRRK2 and FAK occurred mainly in the cytoplasm of microglia and HEK 293T cells (Fig. 6a,b; Supplementary Figs 5,6). Interestingly, this interaction showed little change in response to ADP treatment (Figs $6 \mathrm{~b}$ and $7 \mathrm{a}$; Supplementary Figs $5 \mathrm{~b}$ and $6 \mathrm{a}$ ) or the LRRK2 mutation 
a

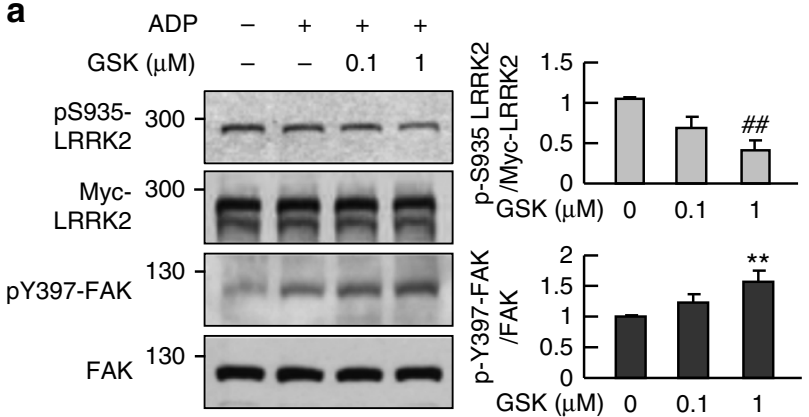

b
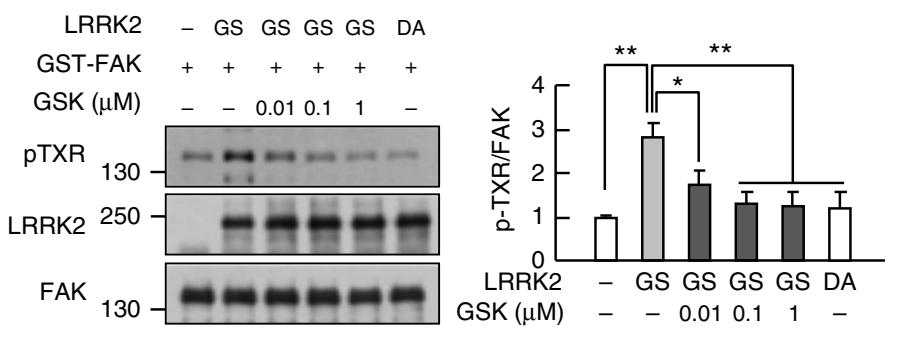

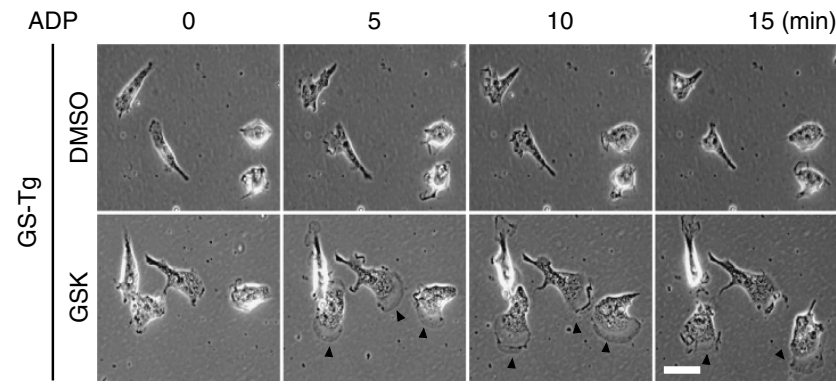

d
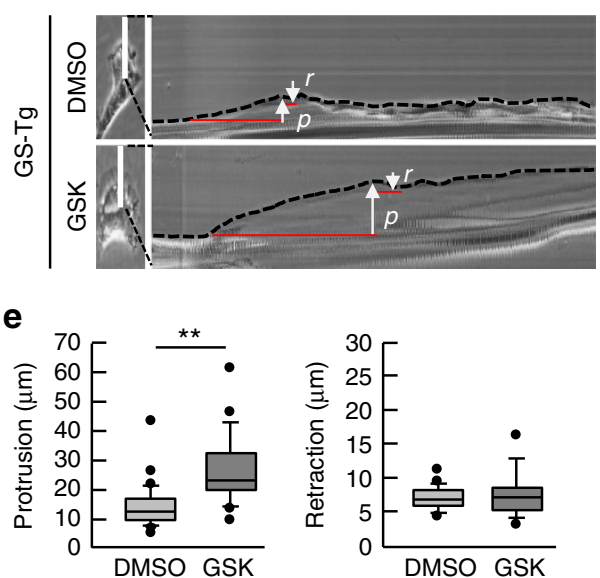

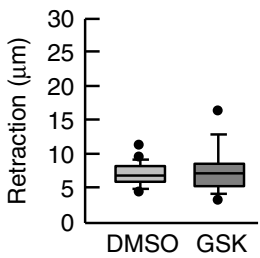

Figure 8 | The LRRK2 kinase inhibitor, GSK2578215A (GSK), reduces pTXR-FAK levels and rescues pY397-FAK levels and motility of GS-Tg microglia. (a) HEK 293 T cells expressing GS-LRRK2 were treated with ADP $(100 \mu \mathrm{M})$ for $5 \mathrm{~min}$ in the presence of the indicated amount of GSK. Levels of S935autophosphorylated LRRK2 (pS935-LRRK2) and Y397-autophosphorylated FAK ( $p$ Y397-FAK) were measured by western blot using antibodies specific for pS935-LRRK2 and pY397-FAK, respectively. FAK and Myc were used as loading controls. Band intensities were quantified and plotted. Values are means \pm s.e.m. of three separate experiments. (b) In vitro kinase assays were carried out using recombinant GST-FAK and GS-LRRK2 (GS) or DA-LRRK2 (DA) in the absence or presence of the indicated amount of GSK. pTXR-FAK was analysed and plotted. Values are means \pm s.e.m. of three separate experiments. (c-e) GS-Tg microglia were treated with GSK $(1 \mu \mathrm{M})$ for 30 min and then treated with ADP. ADP $(100 \mu \mathrm{M})$ induced formation of stable lamellipodia followed by cell body movement in the presence of GSK (c) arrowheads). SACED showed that GSK $(n=26)$ increased protrusion compared to DMSO ( $n=32$ ) (d,e). One-way ANOVA with Newman-Keuls post hoc test, ${ }^{\star} P<0.05,{ }^{\star \star} P<0.01,{ }^{\# \#} P<0.01$ in $\mathbf{a}$ and $\mathbf{b}$. Two-tailed Student's $t$-test, ${ }^{\star \star} P<0.01$ in $\mathbf{e}$. Scale bar, $50 \mu \mathrm{m}$ (c). ANOVA, analysis of variance.

(Figs $6 \mathrm{~b}$ and $7 \mathrm{~b}$ ). In HEK 293T cells, however, the number of focal adhesions increased following ADP treatment (Fig. 7c,d); in the latter system, the GS and DA mutations, respectively, decreased and increased the number of focal adhesions compared with WT LRRK2 (Fig. 7d). Furthermore, when HEK cells were plated on fibronectin, LRRK2 was not located at focal adhesion strongly stained with FAK and paxillin (Supplementary Fig. 6b). Accordingly, FAK but not LRRK2 was located at the edge of lamellipodia (Fig. 6a; Supplementary Fig. 5a). On the basis of these results, we speculate that FAK free from LRRK2-mediated phosphorylation was phosphorylated on Y397 and positively regulates microglia motility and/or focal adhesion of HEK 293T cells (Fig. 9).

Next arising question was what is a physiological role for LRRK2 in modulating microglial motility. We speculate that LRRK2 inhibits unnecessary movement of cells, since LRRK2-KD BV2 cells were highly motile even in the absence of a stimulus (Fig. 1). Although continuous surveillance in the brain is an important microglial function ${ }^{12-14}$, excessive movement would entail the expenditure of unnecessary energy and effort. Therefore, LRRK2 may balance the surveillance function of microglia with the competing need to conserve energy. However, LRRK2 mutations such as the gain-of-function G2019S excessively inhibited microglial surveillance function (Figs 1 and 2), which may result in an inadequate response of microglia to brain injury and the accumulation of defects in the brain.

In conclusion, LRRK2 G2019S mutation leads to defects in microglia motility and response to injury. Studies on PD and other neurodegenerative diseases have focused on neurons. However, neuronal death can be induced not only by defects in neurons themselves but also by defects in glia including microglia, which support neuronal survival and function in diverse ways. Since PD-related genes such as LRRK2, DJ-1, PINK1 and Parkin are expressed in glia, mutations or knockout of these genes alter functions of glia ${ }^{22,65-68}$. Our present findings that the pathogenic mutant, LRRK2 G2019S, causes defects in microglial motility are consistent with the microglial defects clearly observed in other neurodegenerative diseases, including Alzheimer's disease and Huntington's disease ${ }^{18,19}$.

\section{Methods}

Animals. G2019S-LRRK2-Tg FVB mice ${ }^{69}$ were purchased from Jackson Laboratory (stock \#009609, Bar Harbor, ME, USA). Cx3crl-GFP (GFP/GFP) mice were kindly gifted from Dr Seog Bae Oh (Seoul National University School of Dentistry, Seoul, Korea). Non-Tg and G2019S-LRRK2-Tg heterozygote mice were prepared by crossing G2019S-LRRK2 heterozygote mice with wild-type FVB mice or Cx3cr1GFP (GFP/GFP) mice ${ }^{19}$. Genotyping was carried out as described in manufacturer's instruction (Jackson Laboratory). All animal procedures were approved by the Ajou University Institutional Animal Experimentation Committee (AMC-119). 


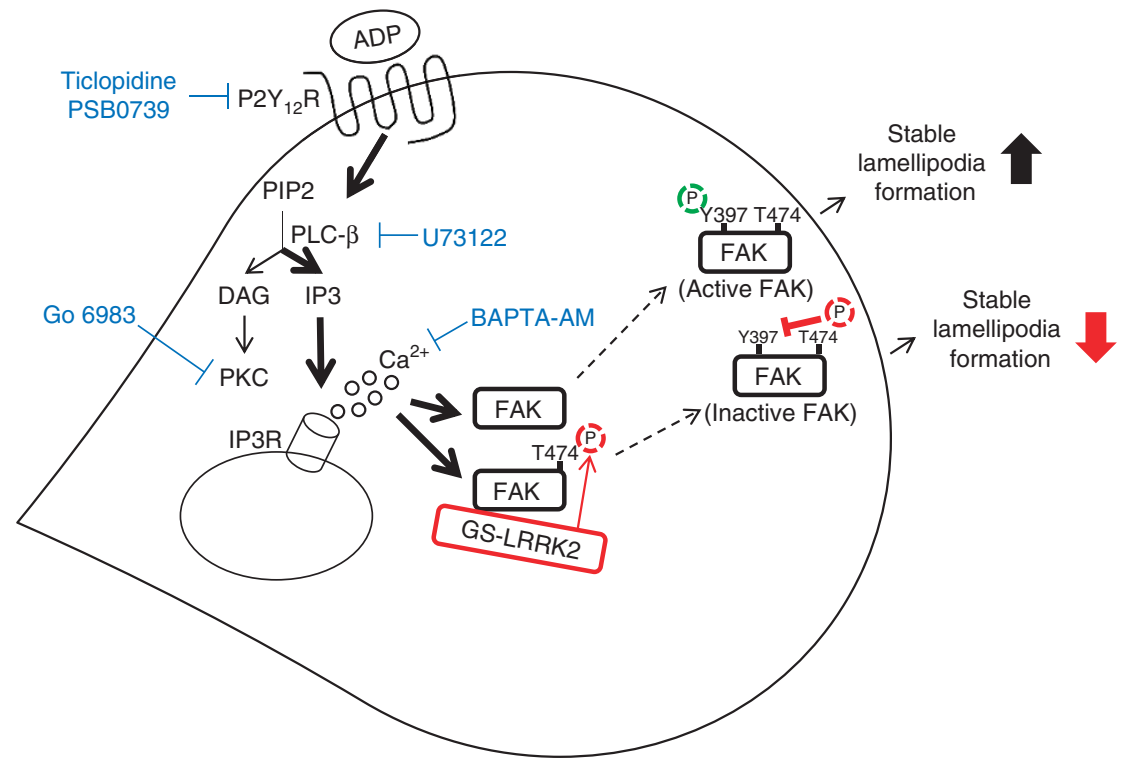

Figure 9 | LRRK2 regulates microglial motility through inhibition of focal adhesion kinase. In response to ADP, microglia rapidly form lamellipodia and become highly motile. In this process, ADP activates FAK (demonstrated by $\mathrm{Y}_{397}$ phosphorylation) by activation of $\mathrm{P}_{2} \mathrm{Y}_{12}$ receptors and their downstream PLC- $\beta$, and intracellular $\mathrm{Ca}^{2}+$ release. Activated pY397-FAK is localized to leading edge of the cell and induces stable lamellipodia formation for proper cell migration. LRRK2 directly interacts and phosphorylates FAK on T474 in TXR motif, which prevents phosphorylation of FAK Y397. GS-LRRK2 excessively inhibits FAK Y397 phosphorylation with its enhanced kinase activity, resulting in unstable lamellipodia formation and improper migration.

Cell culture. Primary microglia were obtained from mixed glia cultured from cerebral cortices of 1-day-old Sprague Dawley rats (Samtako, Seoul, Korea), or wild type and G2019S-LRRK2 heterozygote mice as described previously ${ }^{70}$. Genotyping was carried out as described in manufacturer's instruction (Jackson Laboratory). Briefly, cortices were isolated and single-cell suspensions were prepared by triturating with Pasteur pipettes in Minimal Essential Medium (Sigma, St Louis, MO, USA) containing penicillin/streptomycin $\left(100 \mathrm{U} \mathrm{ml}^{-1}\right), 10 \mathrm{mM}$ HEPES, $10 \%$ fetal bovine serum (FBS; HyClone), and $2 \mathrm{mM}$ L-glutamine. Cells were plated at $75 \mathrm{~cm}^{2}$ T-flask (BD Bioscience, San Jose, CA, USA) and incubated for 2-3 weeks. Microglia were detached from flasks by shaking and then filtered through nylon mesh to remove other cells and cell clumps. Microglia were counted and plated onto culture dishes at an appropriate density. The BV2 murine microglia cell lines were grown in DMEM containing $4 \mathrm{mM}$ L-glutamine, $20 \mathrm{mM}$ HEPES (pH 7.4), penicillin/streptomycin $\left(50 \mathrm{U} \mathrm{ml}^{-1}\right)$ and 10\% (v/v) FBS. BV2 clones LRRK2-KD and NT, stably expressing shRNA targeting LRRK2 (sc-45750-V; Santa Cruz Biotechnology, Santa Cruz, CA, USA) or non-targeting shRNA controls (sc108080; Santa Cruz Biotechnology), respectively, were prepared by transfection with lentiviral particles and subsequent selection ${ }^{22}$. In detail, cells $\left(5 \times 10^{4}\right.$ cells per well) were seeded in 12-well plates and incubated with polybrene $\left(5 \mu \mathrm{g} \mathrm{ml}^{-1}\right)$ and lentiviral particles $\left(1.0 \times 10^{5}\right.$ infectious units) for $12 \mathrm{~h}$. Infected cells were selected with puromycin $\left(5 \mu \mathrm{g} \mathrm{ml}^{-1}\right)$ for 2 days, and plated into 96 -well plates at a density of 0.5 cells per well for clonal selection with puromycin. LRRK2-KD clones that expressed $<20 \%$ of basal levels of Lrrk2 messenger RNA were chosen. The HEK $293 \mathrm{~T}$ cell line was maintained in DMEM supplemented with 10\% FBS.

DNA constructs. Plasmid DNA for C-terminal 3xMyc-tagged wild-type LRRK2 (WT-LRRK2), G2019S-LRKK2 and D1994A-LRKK2 were kind gifts from Dr Wongi Seol (Wonkwang University, Gyeonggi-do, South Korea). FLAG-FAK was prepared by inserting coding sequences of the human FAK gene (NM_153831.3) into the p3xFLAG-CMV-7.1 vector (Sigma) using AccuPrime Pfx DNA Polymerase (Invitrogen, Carlsbad, CA, USA) and an infusion cloning kit (Clontech, Palo Alto, CA, USA). Inserts for FLAG-FAK fragments (D1-D4) and FLAGLRRK2 fragment were prepared from FLAG-FAK and Myc-tagged WT LRRK2 vectors, respectively. Mutations were introduced into FLAG-FAK using a QuikChange Lightning Site-Directed Mutagenesis Kit (Agilent Technologies, Palo Alto, CA, USA). Primers used for mutations are listed in Supplementary Table 1.

Transfection. HEK 293T cells were transfected with DNA plasmids using the jetPEI transfection reagent (Polyplus-Transfection, San Diego, CA, USA) as described by the manufacturer. Briefly, cells were exposed to DNA plasmids and jetPEI mixture for $4 \mathrm{~h}$. Media were then replaced with fresh DMEM containing $10 \%$ FBS. Two days later, transfected cells were used for experiments.

Time-lapse microscopy and SACED. Time-lapse images were obtained every $5 \mathrm{~s}$ for 15-20 min on an inverted microscope (Zeiss Xiovert 200) equipped with a $\times 20$ objective. Stroboscopic images were generated in a $5 \times 200$-pixel-wide $(5 \mathrm{~s} \times 147$ $\mathrm{m})$ box drawn in the direction of cell protrusions using Metamorph software (Nashville, TN, USA). Box and whisker plots for the protrusion distance $(p)$ and retraction distance $(r)$ of individual events $(n>20)$ were produced using SigmaPlot software. The middle line in each box indicates the median; the top of each box indicates the 75th percentile; the bottom indicates the 25th percentile; and the whiskers indicate the extent of the 5th and 95th percentiles.

Migration assay. Microglia $\left(10^{5}\right.$ cells per well) were seeded onto 8 - $\mu \mathrm{m}$-pore Transwells (Corning, Inc., Lowell, MA, USA), and the Transwells were placed on a chamber containing $100 \mu \mathrm{M}$ ADP. Cells were allowed to migrate for $4-12 \mathrm{~h}$. Nonmigrated cells were removed from the top surface of Transwells with a cotton swab. Cells that had migrated to the bottom surface of the Transwells were fixed with $4 \%$ paraformaldehyde for $20 \mathrm{~min}$ and visualized by staining with $1 \%$ Crystal violet (Sigma). The number of migrated cells in nine randomly chosen fields was counted. The FAK inhibitors, FAK 14 and PF573228 (Santa Cruz), were treated for $30 \mathrm{~min}$ before adding cells on top surface.

In vivo stab-wound injury model and preparation of tissues. Male non-Tg and G2019S-LRRK2-Tg heterozygote mice (10 weeks old) were anaesthetized by Avertin (Sigma) and positioned in a stereotaxic apparatus (Kopf Instruments, Tujunga, CA, USA). Stab wound was produced with a $26-\mathrm{G}$ needle in the right striatum (AP, $-1.3 \mathrm{~mm}$; ML, $-1.2 \mathrm{~mm}$; DV, $-3.8 \mathrm{~mm}$ from the bregma) according to the atlas of Paxinos and Franklin. At $1 \mathrm{~h}$ after the injury, mice were anaesthetized and transcardially perfused with saline solution containing $0.5 \%$ sodium nitrate and heparin $\left(10 \mathrm{U} \mathrm{ml}^{-1}\right)$, and then with $4 \%$ paraformaldehyde in $0.1 \mathrm{M}$ phosphate buffer ( $\mathrm{pH}$ 7.4). Brains were obtained and post-fixed overnight at $4^{\circ} \mathrm{C}$ in $4 \%$ paraformaldehyde. Fixed brains were stored at $4{ }^{\circ} \mathrm{C}$ in a $30 \%$ sucrose solution until they sank. Series of coronal sections $(30 \mu \mathrm{m})$ were obtained with a cryostat (Leica, Wetzlar, Germany), and used for immunohistochemistry.

Two-photon microscopic analysis. Mice expressing GFP in their microglia were obtained by crossing G2019S-LRRK2 heterozygous mice (GS/-) with Cx3cr1-GFP $(G F P / G F P)$ mice. For open-skull craniotomy surgery, mice were anaesthetized with Zoletil 50 (Virbac, intramuscular injection, $30 \mu \mathrm{l}$ ), fixed on the stereotactic heating plate (Live Cell Instruments, Seoul, Korea). After removing the scalp and the periosteum, the region ( $1 \mathrm{~mm}$ from bregma, $1 \mathrm{~mm}$ from sagittal suture; diameter, $3 \mathrm{~mm}$ ) of the skull was drilled with a microdrill. Then, the bone was removed elaborately. Three-millimetre-round coverslip was attached to the region with Loctite 454 (Loctite, Rock Hill, CT, USA). Last, emerged portions of the skull were covered with dental acryl. Multiphoton imaging was performed using a LSM 7 MP two-photon laser-scanning microscope (Carl Zeiss Microscopy GmbH, Oberkochen, Germany $)^{71}$. Briefly, laser (920-nm wavelength, $30-\mathrm{mW}$ intensity) was transiently applied to the brain (135- $\mu \mathrm{m}$ depth from the surface). The areas uncovered by microglia were measured, and plotted time-dependent changes in the 
area. Image Analysis Software (Perkin-Elmer, Waltham, MA, USA) was used to process the images and track the movements of microglia.

Immunostaining. For 3,3'-diaminobenzidine staining, serial sections were rinsed three times with PBS, treated with $3 \% \mathrm{H}_{2} \mathrm{O}_{2}$ for $5 \mathrm{~min}$ and rinsed with PBS containing $0.2 \%$ Triton X-100 (PBST). Non-specific binding was blocked with $1 \%$ bovine serum albumin in PBST. Sections were incubated overnight at room temperature with primary antibodies specific for Iba-1 (1:1,000, 019-19741, Wako). Following rinsing in PBST, sections were incubated with biotinylated secondary antibodies (Vector Laboratories, Burlingame, CA), and visualized according to the manufacturer's guidance. Sections were mounted on gelatin-coated slides, and examined under a bright-field microscope (Olympus Optical, BX51, Tokyo, Japan). Images were analysed using Image J (NIH, Bethesda, MD, USA).

For immunofluorescence staining, cells were seeded onto coverslips (Fisher Scientific Co., Fair Lawn, NJ, USA), fixed with $4 \%$ paraformaldehyde in a cytoskeleton stabilization buffer (10 mM MES ( $\mathrm{pH} 6.1$ ), $138 \mathrm{mM} \mathrm{KCl}, 3 \mathrm{mM}$ $\mathrm{MgCl}_{2}, 2 \mathrm{mM}$ EGTA and $0.32 \mathrm{M}$ sucrose), permeabilized with $0.1 \%$ Triton X-100 and treated with $1 \%$ bovine serum albumen. Cells were incubated with primary antibodies against LRRK2 (1:50, N231B/34 and N241 A/34, NeuroMab), FAK (1:50, \#3285, Cell Signaling), pY397-FAK (1:20, \#3283, Cell Signaling), Myc-tag (1:500, \#2278, Cell Signaling) and GFP (1:500, ab13970, Abam), and visualized with Alexa488- and/or Alexa-555-conjugated secondary antibodies (1:500, A21202 and A31572, Invitrogen). Cells were incubated with Phalloidin-conjugated Alexa-488 ( $30 \mathrm{nM}$; Cytoskeleton Inc., Denver, CO, USA) used for F-actin staining. Cells were mounted with Vecta shield (Vector Laboratories). Images were captured with a confocal microscope (LSM510 Carl Zeiss, Oberkochen, Germany).

Proximity ligation assay. Cells were fixed with $4 \%$ paraformaldehyde, permeabilized with $0.1 \%$ Triton X-100 and treated with $1 \%$ bovine serum albumen. Cells were then incubated with LRRK2 (1:50, N231B/34 and N241A/34, NeuroMab) and FAK (1:50, \#3285, Cell Signaling) antibodies together and incubated with DNA probe-conjugated secondary antibodies (Olink Bioscience, Uppsala, Sweden) for $1 \mathrm{~h}$ at $37^{\circ} \mathrm{C}$. Cells were washed, and DNA probes were ligated for $30 \mathrm{~min}$ at $37^{\circ} \mathrm{C}$, amplified for $2 \mathrm{~h}$ at $37^{\circ} \mathrm{C}$, and examined under an Axiovert $200 \mathrm{M}$ microscope (Carl Zeiss). Images were analysed using Image J (NIH, Bethesda, MD, USA).

Western blot analysis. Cells and mouse brains were lysed on ice in RIPA buffer (50 mM Tris- $\mathrm{HCl}$ (pH 7.4), 1\% NP-40, $1 \mathrm{mM} \mathrm{NaF}, 0.25 \%$ Na-deoxycholate, $1 \mathrm{mM}$ $\mathrm{Na}_{3} \mathrm{VO}_{4}$ and $150 \mathrm{mM} \mathrm{NaCl}$ ) containing protease/phosphatase inhibitor cocktail (GenDEPOT, Barker, TX, USA). Lysates were centrifuged, and proteins in the supernatant were separated by SDS-polyacrylamide gel electrophoresis and blotted onto nitrocellulose membranes (Protran, Schleicher \& Schuell, Dassel, Germany). Membranes were incubated with antibodies specific for LRRK2 (1:1,000, ab133474, Abcam), pS935-LRRK2 (1:1,000, ab133450, Abcam), FAK (1:1,000, \#3285, Cell Signaling), pY397-FAK (1:1,000, \#3283, Cell Signaling), Myc (1:1,000, \#2278, Cell Signaling), FLAG (1:1,000, F1804, Sigma), pTXR (1:1,000, \#2351, Cell Signaling) and glyceraldehyde-3-phosphate dehydrogenase (GAPDH, 1:1,000, sc-48167, Santa Cruz). Membranes were washed with Tris-buffered saline containing $0.1 \%$ Tween 20 , incubated with secondary antibodies and visualized with an enhanced chemiluminescence system (Daeil Lab Inc., Seoul, Korea). Uncropped scan for the main figures is presented in Supplementary Fig. 10.

Immunoprecipitation assay. Cells and brain tissue were lysed with an immunoprecipitation buffer (1\% Triton X-100, $150 \mathrm{mM} \mathrm{NaCl}, 10 \mathrm{mM} \mathrm{NaH} \mathrm{PO}_{4}$, $15 \mathrm{mM} \mathrm{Na}_{2} \mathrm{HPO}_{4}, 50 \mathrm{mM} \mathrm{NaF}, 1 \mathrm{mM}$ EDTA and $1 \mathrm{mM} \mathrm{Na}_{3} \mathrm{VO}_{4}$ ). Human brain lysates were purchased from Novus Biological (Littleton, CO, USA). Cell lysates and mouse and human brain lysates $(200-500 \mu \mathrm{g})$ were incubated with primary antibodies against LRRK2 $(1 \mu \mathrm{g}$, ab181386, Abcam), FAK (1 $\mu \mathrm{g}$, sc-558, Santa Cruz), FLAG (1 $\mu \mathrm{g}, \mathrm{F} 1804$, Sigma) and IgG (1 $\mu \mathrm{g}, \# 3900$, Cell Signaling), and then with Protein $\mathrm{G}$ agarose beads ( $20 \mu \mathrm{l}$ per a reaction, Millipore, Billerica, MA, USA). Beads were washed with immunoprecipitation buffer and boiled in $2 \times$ sample buffer. Immunoprecipitated proteins were identified by western blot.

In vitro kinase assay. For in vitro kinase assays, $50 \mathrm{ng}$ of recombinant human GST-WT-LRRK2, GST-G2019S-LRRK2 or GST-D1994A-LRRK2 (Invitrogen) were incubated with $250 \mathrm{ng}$ of recombinant human GST-FAK (Invitrogen) in kinase buffer S (50 mM Tris- $\mathrm{HCl}(\mathrm{pH} 8.5), 10 \mathrm{mM} \mathrm{MgCl}_{2}, 0.01 \%$ Brij-35 and $1 \mathrm{mM}$ EGTA; Invitrogen) including protease/phosphatase inhibitor cocktail (GenDEPOT), $10 \mu \mathrm{M}$ ATP and/or $1 \mu \mathrm{Ci} \mathrm{ml}^{-1}{ }^{32} \mathrm{P}$-ATP (Perkin-Elmer-Cetus, Norwalk, CT, USA). For some experiments, WT and mutant FLAG-FAK constructs (FAK $(\mathrm{T} \rightarrow \mathrm{E}), \mathrm{K} 454 \mathrm{R}$ or T474A) were expressed in HEK 293T cells, immunoprecipitated, isolated from agarose beads and used for in vitro kinase assays. To release FLAGFAK proteins from agarose beads, the protein-antibody-bead complex was treated with $100 \mu \mathrm{M}$ FLAG peptide (Sigma) in $50 \mathrm{mM}$ Tris- $\mathrm{HCl}$ (pH 7.4).

Image analysis. Band intensities in western blots and Coomassie blue-stained gels were quantified using one-dimensional scan software (Scanalytics, Fairfax, VA, USA).
Statistical analysis. The statistical significance of differences between two groups was determined using unpaired two-tailed Student's $t$-tests. For multiple-means comparisons, statistical significance was determined by one-way analysis of variance followed by Newman-Keuls post hoc test or two-way analysis of variance with Bonferroni post hoc test using Graph Pad Prism 5 (GraphPad Software, CA, USA).

\section{References}

1. Mata, I. F., Wedemeyer, W. J., Farrer, M. J., Taylor, J. P. \& Gallo, K. A. LRRK2 in Parkinson's disease: protein domains and functional insights. Trends Neurosci. 29, 286-293 (2006).

2. Chan, D., Citro, A., Cordy, J. M., Shen, G. C. \& Wolozin, B. Rac1 protein rescues neurite retraction caused by G2019S leucine-rich repeat kinase 2 (LRRK2). J. Biol. Chem. 286, 16140-16149 (2011).

3. Jaleel, M. et al. LRRK2 phosphorylates moesin at threonine-558: characterization of how Parkinson's disease mutants affect kinase activity. Biochem. J. 405, 307-317 (2007).

4. Parisiadou, L. et al. Phosphorylation of ezrin/radixin/moesin proteins by LRRK2 promotes the rearrangement of actin cytoskeleton in neuronal morphogenesis. J. Neurosci. 29, 13971-13980 (2009).

5. Meixner, A. et al. A QUICK screen for Lrrk2 interaction partners--leucine-rich repeat kinase 2 is involved in actin cytoskeleton dynamics. Mol. Cell. Proteomics 10, 001172 (2011).

6. Paisan-Ruiz, C., Washecka, N., Nath, P., Singleton, A. B. \& Corder, E. H Parkinson's disease and low frequency alleles found together throughout LRRK2. Ann. Hum. Genet. 73, 391-403 (2009).

7. Lesage, S. et al. LRRK2 G2019S as a cause of Parkinson's disease in North African Arabs. N. Engl. J. Med. 354, 422-423 (2006).

8. Ozelius, L. J. et al. LRRK2 G2019S as a cause of Parkinson's disease in Ashkenazi Jews. N. Engl. J. Med. 354, 424-425 (2006).

9. Smith, W. W. et al. Leucine-rich repeat kinase 2 (LRRK2) interacts with parkin, and mutant LRRK2 induces neuronal degeneration. Proc. Natl Acad. Sci. USA 102, 18676-18681 (2005).

10. MacLeod, D. et al. The familial Parkinsonism gene LRRK2 regulates neurite process morphology. Neuron 52, 587-593 (2006).

11. Lee, B. D., Dawson, V. L. \& Dawson, T. M. Leucine-rich repeat kinase 2 (LRRK2) as a potential therapeutic target in Parkinson's disease. Trends Pharmacol. Sci. 33, 365-373 (2012).

12. Nimmerjahn, A., Kirchhoff, F. \& Helmchen, F. Resting microglial cells are highly dynamic surveillants of brain parenchyma in vivo. Science 308, 1314-1318 (2005).

13. Davalos, D. et al. ATP mediates rapid microglial response to local brain injury in vivo. Nat. Neurosci. 8, 752-758 (2005).

14. Li, Y., Du, X. F., Liu, C. S., Wen, Z. L. \& Du, J. L. Reciprocal regulation between resting microglial dynamics and neuronal activity in vivo. Dev. Cell $\mathbf{2 3}$, 1189-1202 (2012).

15. Hines, D. J., Hines, R. M., Mulligan, S. J. \& Macvicar, B. A. Microglia processes block the spread of damage in the brain and require functional chloride channels. Glia 57, 1610-1618 (2009).

16. Jeong, H. K. et al. Inflammatory responses are not sufficient to cause delayed neuronal death in ATP-induced acute brain injury. PLoS One 5, el3756 (2010)

17. Jeong, H. K., Ji, K., Min, K. \& Joe, E. H. Brain inflammation and microglia: facts and misconceptions. Exp. Neurobiol. 22, 59-67 (2013).

18. El Khoury, J. et al. Ccr2 deficiency impairs microglial accumulation and accelerates progression of Alzheimer-like disease. Nat. Med. 13, 432-438 (2007).

19. Kwan, W. et al. Mutant huntingtin impairs immune cell migration in Huntington disease. J. Clin. Invest. 122, 4737-4747 (2012).

20. Damani, M. R. et al. Age-related alterations in the dynamic behavior of microglia. Aging Cell 10, 263-276 (2011).

21. Hefendehl, J. K. et al. Homeostatic and injury-induced microglia behavior in the aging brain. Aging Cell 13, 60-69 (2014).

22. Kim, B. et al. Impaired inflammatory responses in murine Lrrk2-knockdown brain microglia. PLoS One 7, e34693 (2012).

23. Moehle, M. S. et al. LRRK2 inhibition attenuates microglial inflammatory responses. J. Neurosci. 32, 1602-1611 (2012).

24. Lim, S. T. et al. Nuclear FAK promotes cell proliferation and survival through FERM-enhanced p53 degradation. Mol. Cell 29, 9-22 (2008).

25. Yamamoto, D. et al. FAK overexpression upregulates cyclin D3 and enhances cell proliferation via the PKC and PI3-kinase-Akt pathways. Cell. Signal. 15, 575-583 (2003).

26. Mitra, S. K., Hanson, D. A. \& Schlaepfer, D. D. Focal adhesion kinase: in command and control of cell motility. Nat. Rev. Mol. Cell Biol. 6, 56-68 (2005).

27. Dunty, J. M. et al. FERM domain interaction promotes FAK signaling. Mol. Cell. Biol. 24, 5353-5368 (2004).

28. Zheng, C. et al. Differential regulation of Pyk2 and focal adhesion kinase (FAK). The C-terminal domain of FAK confers response to cell adhesion. $J$. Biol. Chem. 273, 2384-2389 (1998). 
29. Kornberg, L. J., Earp, H. S., Turner, C. E., Prockop, C. \& Juliano, R. L. Signal transduction by integrins: increased protein tyrosine phosphorylation caused by clustering of beta 1 integrins. Proc. Natl Acad. Sci. USA 88, 8392-8396 (1991).

30. Guan, J. L., Trevithick, J. E. \& Hynes, R. O. Fibronectin/integrin interaction induces tyrosine phosphorylation of a $120-\mathrm{kDa}$ protein. Cell. Regul. 2, 951-964 (1991).

31. Shyy, J. Y. \& Chien, S. Role of integrins in cellular responses to mechanical stress and adhesion. Curr. Opin. Cell Biol. 9, 707-713 (1997).

32. Luttrell, L. M., Daaka, Y. \& Lefkowitz, R. J. Regulation of tyrosine kinase cascades by G-protein-coupled receptors. Curr. Opin. Cell Biol. 11, 177-183 (1999).

33. Sieg, D. J. et al. FAK integrates growth-factor and integrin signals to promote cell migration. Nat. Cell Biol. 2, 249-256 (2000).

34. Rozengurt, E. Mitogenic signaling pathways induced by G protein-coupled receptors. J. Cell Physiol. 213, 589-602 (2007).

35. Parsons, J. T., Martin, K. H., Slack, J. K., Taylor, J. M. \& Weed, S. A. Focal adhesion kinase: a regulator of focal adhesion dynamics and cell movement. Oncogene 19, 5606-5613 (2000).

36. Haynes, S. E. et al. The P2Y12 receptor regulates microglial activation by extracellular nucleotides. Nat. Neurosci. 9, 1512-1519 (2006)

37. Schaller, M. D. et al. Autophosphorylation of the focal adhesion kinase, pp125FAK, directs SH2-dependent binding of pp60src. Mol. Cell Biol. 14, 1680-1688 (1994).

38. Slack-Davis, J. K. et al. Cellular characterization of a novel focal adhesion kinase inhibitor. J. Biol. Chem. 282, 14845-14852 (2007).

39. Golubovskaya, V. M. et al. A small molecule inhibitor, 1,2,4,5-benzenetetraamine tetrahydrochloride, targeting the y397 site of focal adhesion kinase decreases tumor growth. J. Med. Chem. 51, 7405-7416 (2008).

40. Golubovskaya, V. M. et al. Mitoxantrone targets the ATP-binding site of FAK, binds the FAK kinase domain and decreases FAK, Pyk-2, c-Src, and IGF-1R in vitro kinase activities. Anticancer Agents Med. Chem. 13, 546-554 (2013).

41. Pungaliya, P. P. et al. Identification and characterization of a leucine-rich repeat kinase 2 (LRRK2) consensus phosphorylation motif. PLoS ONE 5, e13672 (2010).

42. Nichols, R. J. et al. Substrate specificity and inhibitors of LRRK2, a protein kinase mutated in Parkinson's disease. Biochem. J. 424, 47-60 (2009).

43. Davies, P. et al. Comprehensive characterization and optimization of antiLRRK2 (leucine-rich repeat kinase 2) monoclonal antibodies. Biochem. J. 453, 101-113 (2013)

44. Reith, A. D. et al. GSK2578215A; a potent and highly selective 2arylmethyloxy-5-substitutent-N-arylbenzamide LRRK2 kinase inhibitor. Bioorg. Med. Chem. Lett. 22, 5625-5629 (2012).

45. Deng, X. et al. Characterization of a selective inhibitor of the Parkinson's disease kinase LRRK2. Nat. Chem. Biol. 7, 203-205 (2011).

46. Ramsden, N. et al. Chemoproteomics-based design of potent LRRK2-selective lead compounds that attenuate Parkinson's disease-related toxicity in human neurons. ACS Chem. Biol. 6, 1021-1028 (2011).

47. Lee, B. D. et al. Inhibitors of leucine-rich repeat kinase-2 protect against models of Parkinson's disease. Nat. Med. 16, 998-1000 (2010).

48. Hall-Jackson, C. A. et al. Paradoxical activation of Raf by a novel Raf inhibitor. Chem. Biol. 6, 559-568 (1999).

49. Ehrenreiter, K. et al. Raf-1 regulates Rho signaling and cell migration. J. Cell Biol. 168, 955-964 (2005).

50. Moehle, M. S. et al. The G2019S LRRK2 mutation increases myeloid cell chemotactic responses and enhances LRRK2 binding to actin-regulatory proteins. Hum. Mol. Genet. 24, 4250-4267 (2015).

51. Caesar, M. et al. Leucine-rich repeat kinase 2 functionally interacts with microtubules and kinase-dependently modulates cell migration. Neurobiol. Dis. 54, 280-288 (2013).

52. Etienne-Manneville, S. Actin and microtubules in cell motility: which one is in control? Traffic 5, 470-477 (2004).

53. Ilic, D. et al. Reduced cell motility and enhanced focal adhesion contact formation in cells from FAK-deficient mice. Nature 377, 539-544 (1995).

54. Leu, T. H. \& Maa, M. C. Tyr-863 phosphorylation enhances focal adhesion kinase autophosphorylation at Tyr-397. Oncogene 21, 6992-7000 (2002).

55. Calalb, M. B., Polte, T. R. \& Hanks, S. K. Tyrosine phosphorylation of focal adhesion kinase at sites in the catalytic domain regulates kinase activity: a role for Src family kinases. Mol. Cell. Biol. 15, 954-963 (1995).

56. Deramaudt, T. B. et al. FAK phosphorylation at Tyr-925 regulates cross-talk between focal adhesion turnover and cell protrusion. Mol. Biol. Cell 22, 964-975 (2011)

57. Calalb, M. B., Zhang, X., Polte, T. R. \& Hanks, S. K. Focal adhesion kinase tyrosine-861 is a major site of phosphorylation by Src. Biochem. Biophys. Res. Commun. 228, 662-668 (1996).

58. Tilghman, R. W. et al. Focal adhesion kinase is required for the spatial organization of the leading edge in migrating cells. J. Cell Sci. 118, 2613-2623 (2005).
59. Xie, Z., Sanada, K., Samuels, B. A., Shih, H. \& Tsai, L. H. Serine 732 phosphorylation of FAK by Cdk5 is important for microtubule organization, nuclear movement, and neuronal migration. Cell 114, 469-482 (2003).

60. Smith, W. W. et al. Kinase activity of mutant LRRK2 mediates neuronal toxicity. Nat. Neurosci. 9, 1231-1233 (2006).

61. Liu, E., Cote, J. F. \& Vuori, K. Negative regulation of FAK signaling by SOCS proteins. EMBO J. 22, 5036-5046 (2003).

62. Angers-Loustau, A. et al. Protein tyrosine phosphatase-PEST regulates focal adhesion disassembly, migration, and cytokinesis in fibroblasts. J. Cell Biol. 144, 1019-1031 (1999).

63. Manes, S. et al. Concerted activity of tyrosine phosphatase SHP-2 and focal adhesion kinase in regulation of cell motility. Mol. Cell. Biol. 19, 3125-3135 (1999).

64. Zheng, Y. et al. FAK phosphorylation by ERK primes ras-induced tyrosine dephosphorylation of FAK mediated by PIN1 and PTP-PEST. Mol. Cell 35 11-25 (2009).

65. Choi, I. et al. Pink1 deficiency attenuates astrocyte proliferation through mitochondrial dysfunction, reduced akt and increased p38 mapk activation, and downregulation egfr. Glia 61, 800-812 (2013).

66. Kim, J. H. et al. DJ-1 facilitates the interaction between STAT1 and its phosphatase, SHP-1, in brain microglia and astrocytes: a novel antiinflammatory function of DJ-1. Neurobiol. Dis 60C, 1-10 (2013).

67. Kim, K. S. et al. DJ-1 associates with lipid rafts by palmitoylation and regulates lipid rafts-dependent endocytosis in astrocytes. Hum. Mol. Genet 22, 4805-4817 (2013).

68. Kim, J. H. et al. Suppression of miR-155 expression in IFN-?-Treated astrocytes and microglia by DJ-1: a possible mechanism for maintaining SOCS1 expression. Exp. Neurobiol 23, 148-154 (2014).

69. Li, Y. et al. Mutant LRRK2(R1441G) BAC transgenic mice recapitulate cardinal features of Parkinson's disease. Nat. Neurosci. 12, 826-828 (2009).

70. Pyo, H., Jou, I., Jung, S., Hong, S. \& Joe, E. H. Mitogen-activated protein kinases activated by lipopolysaccharide and beta-amyloid in cultured rat microglia. Neuroreport 9, 871-874 (1998).

71. Baik, S. H. et al. Migration of neutrophils targeting amyloid plaques in Alzheimer's disease mouse model. Neurobiol. Aging 35, 1286-1292 (2014).

\section{Acknowledgements}

This work was supported by a grant (NRF-2014R1A2A2A01005947) funded by the Korean government (MEST) and a grant (NRF-2012R1A5A2048183) from KOSEF through the Chronic Inflammatory Disease Research Center (CIDRC) at Ajou University (to E-H J), and a grant (NRF-2011-0030049) funded by the Korean government (MSIP) (to HS). We thank to professors Eun Young Kim, Sang Yoon Lee, Yong-Joon Chwae (Ajou University), Byung-Dae Lee (Kyunghee University) and Hyuk Wan Ko (Dongguk University, Seoul, Korea) for helpful discussion.

\section{Author contributions}

E.-H.J. conceived, designed, supervised the project and wrote the paper; I.C. designed, performed and analysed most of the experiments and wrote the paper; B.K. initially found LRRK2-mediated regulation of microglial morphology and movement; J.-W.B and J.-H.K. performed animal surgery and time-lapse imaging; S.H.B. and I.M.-J. performed two-photon live imaging of microglia; Y.H.H. and W.K.S. performed SACED analysis; J.-H.S. performed western blot using human brain lysates; H.S. provided G2019S-Tg mice and midbrain lysates; I.J., S.M.P., Y.-H.S. and H.C.K. designed experiments to examine interaction between FAK and LRRK2.

\section{Additional information}

Supplementary Information accompanies this paper at http://www.nature.com/ naturecommunications

Competing financial interests: The authors declare no competing financial interests

Reprints and permission information is available online at http://npg.nature.com/ reprintsandpermissions/

How to cite this article: Choi, I. et al. LRRK2 G2019S mutation attenuates microglial motility by inhibiting focal adhesion kinase. Nat. Commun. 6:8255 doi: 10.1038/ ncomms9255 (2015)

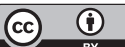

This work is licensed under a Creative Commons Attribution 4.0 International License. The images or other third party material in this article are included in the article's Creative Commons license, unless indicated otherwise in the credit line; if the material is not included under the Creative Commons license, users will need to obtain permission from the license holder to reproduce the material. To view a copy of this license, visit http://creativecommons.org/licenses/by/4.0/ 